\title{
C1q-TNF-related protein-3 attenuates pressure overload-induced cardiac hypertrophy by suppressing the p38/CREB pathway and p38-induced ER stress
}

\author{
Bing Zhang ${ }^{1}$, Ping Zhang ${ }^{1}$, Yanzhen Tan ${ }^{1}$, Pan Feng ${ }^{1}$, Zhengbin Zhang ${ }^{2}$, Hongliang Liang ${ }^{1}$, Weixun Duan ${ }^{1}$,
} Zhenxiao Jin ${ }^{1}$, Xiaowu Wang ${ }^{1}$, Jincheng Liư ${ }^{1}$, Erhe Gao ${ }^{3}$, Shiqiang Yu', Dinghua Yi ${ }^{1}$, Yang Sun ${ }^{1}$ and Wei Yi (1)

\begin{abstract}
C1q-tumor necrosis factor-related protein-3 (CTRP3) is an adipokine, which exerts protective function in ischemic or diabetic heart injury. However, the role of CTRP3 in cardiac hypertrophy remains unclear. The aim of this study was to investigate the pharmacological effects of CTRP3 on pathological cardiac hypertrophy induced by hypertension. Male C57BL/6 J wild-type (WT) mice, Ctrp3 knockout mice, and mice infected with lentivirus overexpressing mouse Ctrp3 underwent sham surgery or transverse aortic constriction (TAC) surgery. After 4 weeks, cardiac hypertrophy, fibrosis, and cardiac function were examined. Compared with WT mice, Ctrp3 deficiency substantially impaired contractile dysfunction, exacerbated the enlargement of cardiomyocytes and myocardial fibrosis, and reprogramed the expression of pathological genes after TAC. Conversely, CTRP3 overexpression played a role in restoring the left ventricular cardiac contractile function, alleviating cardiac hypertrophy and fibrosis, and inhibiting the expression of hypertrophic and fibrotic signaling in mice after TAC. Furthermore, CTRP3 regulated the expression of the p38/CREB pathway and of the primary modulating factors of the endoplasmic reticulum stress, i.e., GRP78 and the downstream molecules eukaryotic translation inhibition factor 2 submit a, C/EBP homologous protein, and inositol-requiring enzyme-1. Further, inhibition of p38 MAPK by SB203580 blunted the ER stress intensified by Ctrp3 deficiency. In vitro, CTRP3 protected neonatal rat cardiac myocytes against phenylephrine-induced cardiomyocyte hypertrophy. We conclude that CTRP3 protects the host against pathological cardiac remodeling and left ventricular dysfunction induced by pressure overload largely by inhibiting the p38/CREB pathway and alleviating p38-induced ER stress.
\end{abstract}

\section{Introduction}

Continuous hypertrophic stimulus such as pressure overload, ischemia, inflammation, and oxidative stress gradually converts the compensatory response into an

\footnotetext{
Correspondence: Dinghua Yi (yidh@fmmu.edu.cn) or

Yang Sun (dr_yangsun@163.com) or Wei Yi (yiwei@fmmu.edu.cn)

'Department of Cardiovascular Surgery, Xijing Hospital, The Fourth Military

Medical University, 127 Changle West Road, 710032 Xi'an, China

${ }^{2}$ Department of Geriatrics, Xijing Hospital, The Fourth Military Medical

University, 127 Changle West Road, 710032 Xi'an, China

Full list of author information is available at the end of the article.

These authors contributed equally: Bing Zhang, Ping Zhang, Yanzhen Tan

Edited by H. Bazan
}

irreversible pathological cardiac hypertrophy, leading to abnormal expression of the cardiac fetal genes, fibrotic reconstruction of the extracellular matrix, and contractile dysfunction, inescapably progressing to the terminal-stage heart failure ${ }^{1-3}$. Currently, there is no effective drug therapy to reverse the progress from pathological cardiac hypertrophy to heart failure ${ }^{4}$. A widely accepted strategy is positive intervention during the early stage of heart failure, inhibiting the pathological cardiac hypertrophy ${ }^{5}$. Regardless of the continuously developing diagnosis and therapeutic approaches, heart failure has still a poor outcome with nearly $25-50 \%$ mortality rate in 5 years

\section{(c) The Author(s) 2019}

(c) (i) Open Access This article is licensed under a Creative Commons Attribution 4.0 International License, which permits use, sharing, adaptation, distribution and reproduction in any medium or format, as long as you give appropriate credit to the original author(s) and the source, provide a link to the Creative Commons license, and indicate if changes were made. The images or other third party material in this article are included in the article's Creative Commons license, unless indicated otherwise in a credit line to the material. If material is not included in the article's Creative Commons license and your intended use is not permitted by statutory regulation or exceeds the permitted use, you will need to obtain permission directly from the copyright holder. To view a copy of this license, visit http://creativecommons.org/licenses/by/4.0/. 
after diagnosis ${ }^{6}$. Therefore, seeking out novel effective therapeutic targets to prevent and alleviate the pathological cardiac hypertrophy is urgent and necessary.

The mitogen-activated protein kinase (MAPK) pathway is closely involved in cardiac hypertrophy ${ }^{7,8}$. MAPKs are major regulatory kinases that directly control numerous physiological processes, including cell proliferation, cell growth, and stress responses. Protein 38 MAPK (p38), one branch of MAPKs, can be activated and phosphorylated under pressure-load stimulation ${ }^{9}$. Numerous groups have studied the role of p38 MAPK in various cardiac hypertrophy settings and showed that pathological cardiac hypertrophy can be suppressed by inhibiting the phosphorylation of $\mathrm{p} 38^{8,10-12}$. The cAMP response elementbinding protein (CREB), a downstream signal molecule of p38, can regulate multiple cellular responses by interacting with DNA and regulating gene transcription ${ }^{13-15}$. Various forms of pathological cardiac hypertrophy can be attenuated by inhibiting the phosphorylation of CREB $^{16,17}$.

A variety of stimuli, such as ischemia, hypoxia, freeradical exposure, elevated protein synthesis, and gene mutations, can interfere with the endoplasmic reticulum (ER) function, leading to the accumulation of unfolded and misfolded proteins in the ER. Consequently, multiple ER transmembrane sensors are activated, generating unfolded protein response ${ }^{18,19}$. A long-term nonresolved or excessive ER stress eventually activates the apoptotic signaling pathway. Further, cardiomyocyte apoptosis induced by the ER stress plays an important role in the transition from cardiac hypertrophy to heart failure, and cardiac hypertrophy can be attenuated by alleviating ER stress ${ }^{18,20,21}$. Therefore, ER stress may be an important intervention target to improve pathological myocardial hypertrophy.

C1q/tumor necrosis factor-related protein-3 (CTRP3) is a member of the CTRP superfamily ${ }^{22,23}$. Studies from other groups and our own groups confirmed that CTRP3 exerts a protective effect against myocardial ischemia reperfusion injury, plays a role in the protective response to vascular injury, and can protect the bone marrow mesenchymal stem cells from hypoxic injury ${ }^{22,24-26}$. That is because of the antiapoptotic activity, and vasodilation and angiogenesis effects of CTRP3. Importantly, CTRP3 can alleviate myocardial remodeling and fibrosis in rat after myocardial infarction ${ }^{27}$. Furthermore, CTRP3 attenuates ER stress in mouse with high-fat diet-induced male reproductive dysfunction ${ }^{28}$.

In this study, we investigated the role of CTRP3 in pathological cardiac hypertrophy. CTRP3 deficiencyaggravated cardiac dysfunction, cardiac hypertrophy, and fibrotic remodeling induced by transverse aortic constriction (TAC) in mice. CTRP3 overexpression exerted an opposite effect, and the protective role of CTRP3 was confirmed in vitro. We demonstrate that CTRP3 exerts these effects by inhibiting the p38/CREB signaling pathway and attenuating ER stress. These findings highlight CTRP3 as a therapeutic target for treating pathological myocardial hypertrophy.

\section{Results \\ CTRP3 expression is upregulated in mouse hypertrophic hearts and PE-treated NRCMs}

To determine the role of CTRP3 in cardiac hypertrophy, we have examined the mRNA and protein level of CTRP3 in the heart at 1,2 , and 4 weeks after surgery, respectively. After 1 week of TAC, although protein expression level of CTRP3 was slightly elevated, the change was not significant (Fig. S1a). After 2 and 4 weeks of TAC, CTRP3 protein expression level was significantly increased with time after TAC, in parallel with increased levels of the hypertrophic indices including atrial natriuretic peptide (ANP) and myosin heavy chain $\beta$ ( $\beta$-MHC) (Fig. S1a). However, the mRNA expression level of CTRP3 was significantly increased at 1,2 , or 4 weeks after TAC compared to SHAM group (Fig. S1b). The mRNA and protein level of CTRP3 in PE-treated NRCMs was also detected. After 24 and $48 \mathrm{~h}$ of PE treatment, CTRP3 protein expression was significantly upregulated with time, in parallel with increased levels of ANP and $\beta$-MHC (Fig. S1c). Consistently, CTRP3 mRNA expression was also upregulated (Fig. S1d).

\section{CTRP3 deficiency aggravates cardiac hypertrophy induced by TAC}

To evaluate the function of CTRP3 in pressure-overload cardiac hypertrophy, Ctrp3-KO mouse was generated (Fig. 1a). No obvious exterior and morphological abnormalities were apparent in 8-10-week-old Ctrp3-KO mice compared with the wild-type (WT) mice. Sham or TAC surgery was performed, and 4 weeks later the cardiac hypertrophy model was confirmed by echocardiographic data and histological staining. The heart weight to body weight ratio $(\mathrm{HW} / \mathrm{BW})$, and the lung weight to body weight ratio (LW/BW) were higher in Ctrp3-KO mice after TAC than in WT mice after TAC (Fig. 1b and Fig. S2a). Ctrp3-KO resulted in more severe cardiac dysfunction in TAC-operated mice than in WT mice, as evidenced by decreased ejection fractions (EF)\% and fraction shortening (FS)\%, and increased interventricular septal thickness at end diastole (IVSd) and left ventricular posterior wall thickness at end diastole (LVPWd) in Ctrp3$\mathrm{KO}$ mice subjected to TAC (Fig. 1c-e and Fig. S2b). The representative M-mode echocardiographic images are shown in Fig. S3a. CTRP3 deficiency increased the size of heart in mice after TAC (Fig. 1f). The heart sections were stained with HE, WGA, and Masson stain to analyze the degree of cardiac pathological remodeling. The cardiac 
a

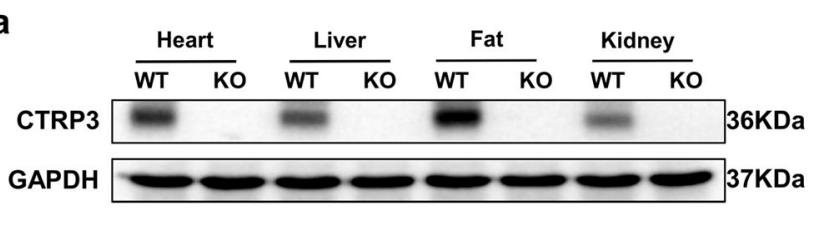

b

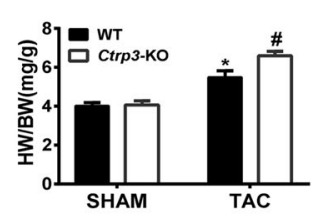

C

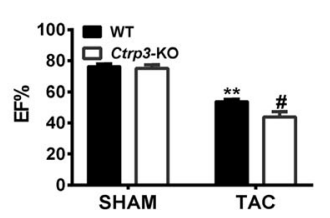

d
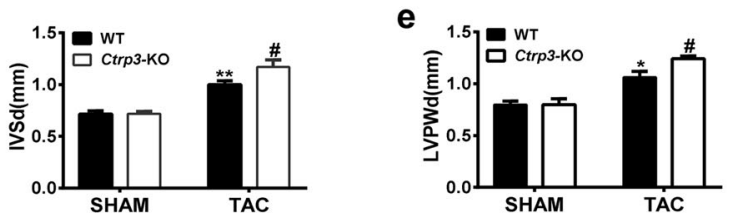

f

$\frac{\text { SHAM }}{\text { WT } \quad \text { Ctrp3-KO }} \frac{\text { TAC }}{\text { WT Ctrp3-Ko }}$
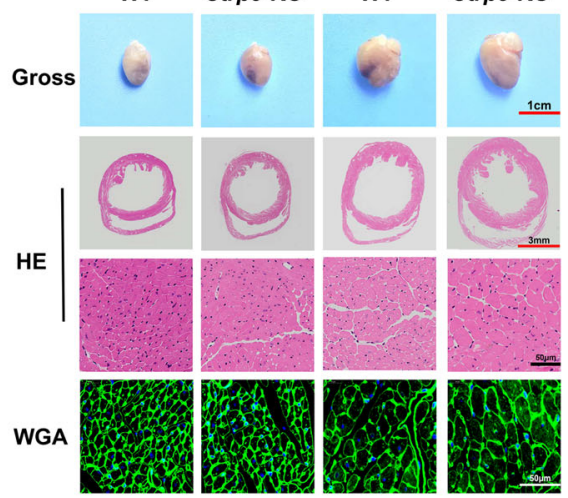

h

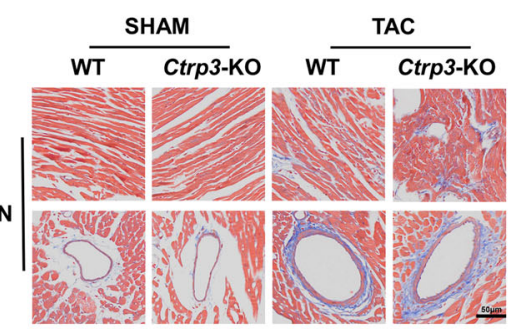

g
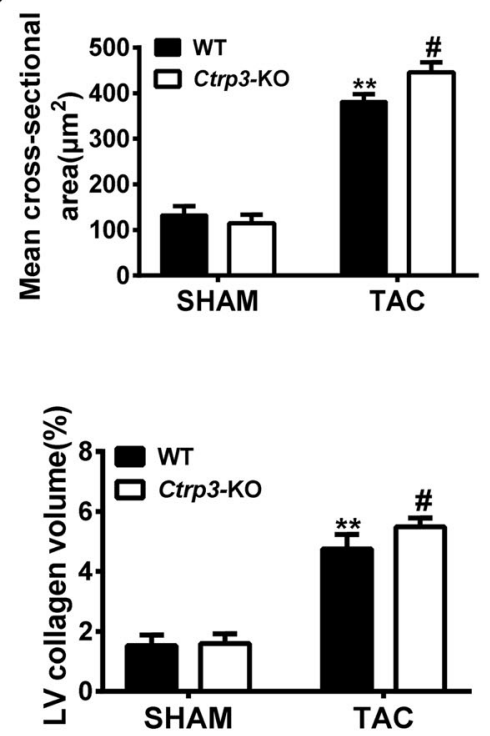

MASSON

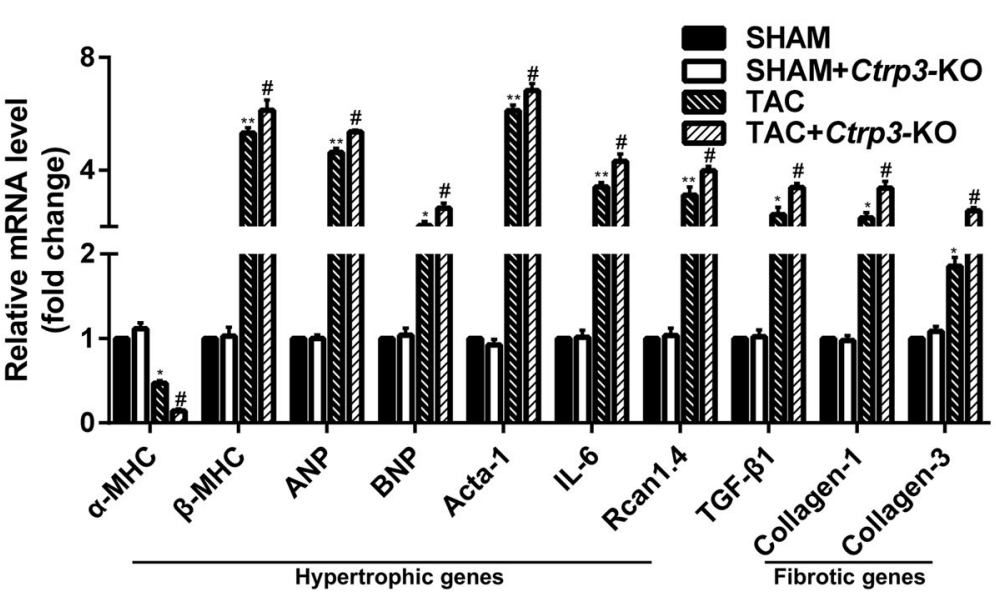

Fig. 1 (See legend on next page.) 
(see figure on previous page)

Fig. 1 CTRP3 deficiency aggravates cardiac hypertrophy induced by TAC. a Representative western blot of CTRP3 levels in different tissues of WT mice and Ctrp3-KO mice ( $n=3$ mice per group). b The HW/BW ratio in animals after 4 weeks of TAC ( $n=5-8$ mice per group). c-e The left ventricular ejection fraction (LVEF), IVSd, and LVPWd, accordingly, determined by analyzing the echocardiographic images ( $n=12-14$ mice per group). $\mathbf{f}$ Representative images of the gross murine heart and sections stained with hematoxylin and eosin (HE), and wheat germ agglutinin (WGA) ( $n=5-8$ mice per group). $\mathbf{g}$ The mean cross-sectional area of cardiomyocytes from the indicated groups ( $n \geq 100$ cells per group). $\mathbf{h}$ Representative images of the murine heart sections (after 4 weeks of TAC) stained with Masson stain, arranged with the perivascular area at the top and the interstitial area at the bottom ( $n=5-8$ mice per group). $\mathbf{i}$ The LV collagen volume in different groups ( $n \geq 40$ fields per group). $\mathbf{j}$ Real-time polymerase chain reaction (real-time PCR) analysis of the expression of genes encoding hypertrophic markers a-MHC, $\beta-M H C$, ANP, BNP, Acta-1, IL-6, and Rcan1.4, and the fibrotic markers TGF- $\beta 1$, collagen-I, and collagen-III in each group ( $n=6$ mice per group). $\mathbf{b}-\mathbf{j}$ The data were analyzed by one-way ANOVA. ${ }^{* *} p<0.01$ vs. SHAM, ${ }^{*} p<0.05$ vs. TAC. In the bar graphs, the data are presented as the mean \pm standard error of the mean (SEM)

hypertrophy and fibrosis of WT mice after TAC were significantly more severe than those in sham-operated WT mice, and CTRP3 deficiency potentiated these responses, as evaluated by determining the average crosssectional area and left ventricular collagen volume (Fig. $1 \mathrm{f}-\mathrm{i})$. Further, after 4 weeks of TAC, the expression of gene for myosin heavy chain $\alpha(\alpha-M H C)$ was significantly downregulated, while that for $\beta-\mathrm{MHC}$ was significantly upregulated in the heart of WT mice compared to shamoperated WT mice (Fig. 1j). CTRP3 deficiency exaggerated this trend (Fig. 1j). The expression of other classic cardiac hypertrophic genes, such as those encoding ANP, brain natriuretic peptide (BNP), $\alpha$-sarcomeric actin, interleukin 6 , and regulator of calcineurin $(\mathrm{CaN}) 1.4$ (Rcan1.4), and fibrotic genes, such as those for the transforming growth factor beta 1 (TGF- $\beta 1$ ), collagen- 1 (Col-1), and collagen-3 (Col-3) were also upregulated in the cardiac tissue of WT mice subjected to TAC compared to the sham group, while CTRP3 deficiency exaggerated this effect (Fig. 1j). The levels of two major downstream regulatory proteins, $\mathrm{CaN}$ and phosphorylated-calmodulin kinase II (CaMKII), were significantly increased in TAC-operated WT mice compared with sham-operated WT mice, and CTRP3 deficiency potentiated this effect (Fig. S4a).

\section{Ctrp3 overexpression represses pressure overload-induced cardiac hypertrophy}

To confirm the protective role of CTRP3 in pressureoverload cardiac hypertrophy, CTRP3 was overexpressed in the cardiac tissue by injection of a lentivirus harboring the Ctrp3 gene (LV-CTRP3) (Fig. 2a). However, as determined by enzyme-linked immunosorbent assay (ELISA), Ctrp3 overexpression in the myocardial tissue was not sufficient to cause a significant increase in serum CTRP3 levels (Fig. 2b). There are no obvious exterior and morphological differences between 8-10-week-old shamoperated WT mice and LV-CTRP3 mice (data not shown). No morphological and functional changes of the heart were observed at baseline, but Ctrp 3 overexpression significantly reversed TAC-induced decrease in the HW/
$\mathrm{BW}$ and $\mathrm{LW} / \mathrm{BW}$, indicating a relief of pulmonary congestion (Fig. 2c and Fig. S2c). Moreover, the abovementioned echocardiography-detectable dysfunction and ventricular dimension enlargement were attenuated in TAC-operated LV-CTRP3 mice compared with WT mice after TAC (Fig. 2d-f, Figs. S2d, S3b). Analysis of the gross heart morphology indicated that Ctrp3 overexpression reduced the heart size in mice after TAC (Fig. 2g). In addition, TAC-triggered cardiac hypertrophic and fibrotic remodeling were blunted in TAC-operated LV-CTRP3 mice compared with WT mice after TAC, as evidenced by reduced average cross-sectional area and left ventricular collagen volume (Fig. $2 \mathrm{~g}-\mathrm{j}$ ). Finally, Ctrp3 overexpression reversed the expression trend of $\alpha-\mathrm{MHC}$ and $\beta-\mathrm{MHC}$ genes, and decreased the degree of upregulation of the above-mentioned marker genes in TAC-operated mice (Fig. 2k). Ctrp3 overexpression also alleviated the activation of CaN and CaMKII (Fig. S4b).

\section{CTRP3 alters phenylephrine (PE)-induced cardiomyocyte hypertrophy in neonatal rat cardiac myocytes (NRCMS)}

To further test the antihypertrophic effect of CTRP3, a PE-induced cardiomyocyte hypertrophy model was established. PE-treated NRCMs were administered CTRP3 or Ctrp3 siRNA (si-CTRP3). The knockdown effect of si-CTRP3 on CTRP3 expression was detected and significant (Fig. S5). We found that CTRP3 depletion exacerbated the PE-induced increase of cell size, and mRNA level of $\beta$-MHC, ANP, and BNP (Fig. 3a, b). Changes in ANP, $\beta-\mathrm{MHC}$, and CaN protein levels were consistent with the immunostaining and gene expression data (Fig. 3c). However, these trends were completely reversed by CTRP3 supplementation, indicating a protective role of CTRP3 against pathological cell growth in vitro (Fig. $3 d-f$ ).

\section{CTRP3 regulates activation of the p38 MAPK/CREB pathway in TAC mouse}

To explore the mechanism of CTRP3-based inhibition of cardiac hypertrophy, several signaling pathways related to the process of hypertrophy were tested. We found that 

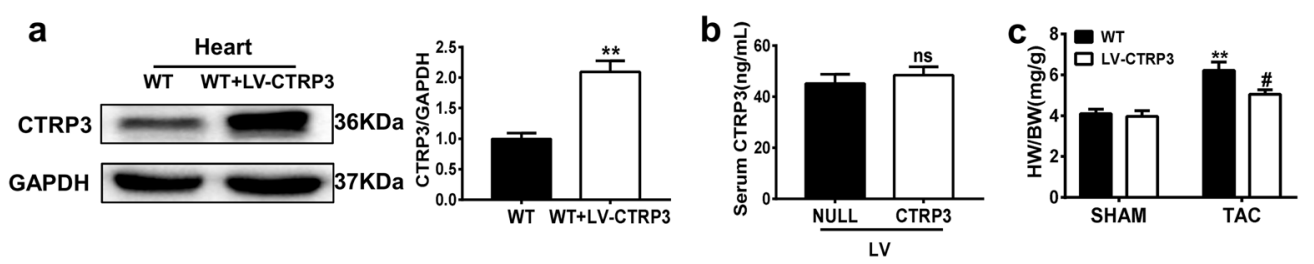

d

e

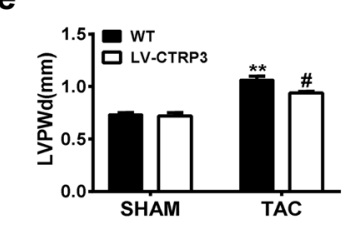

f
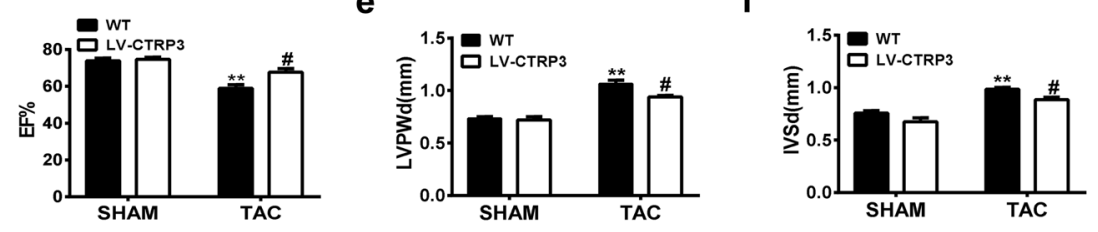

g

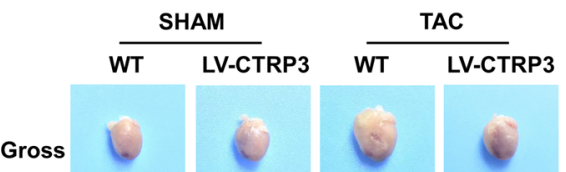

h

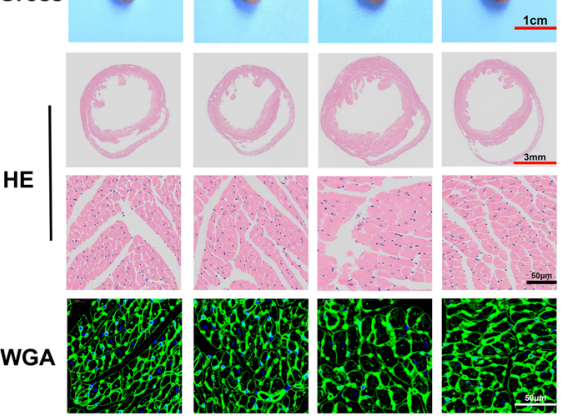

i

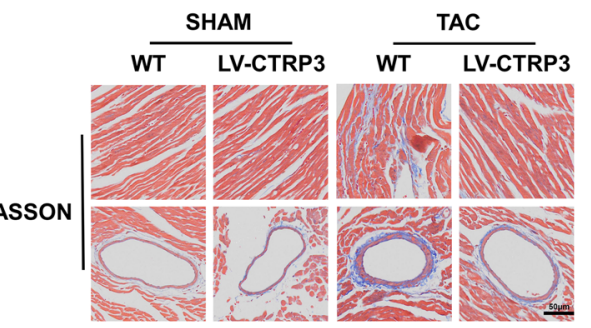

j

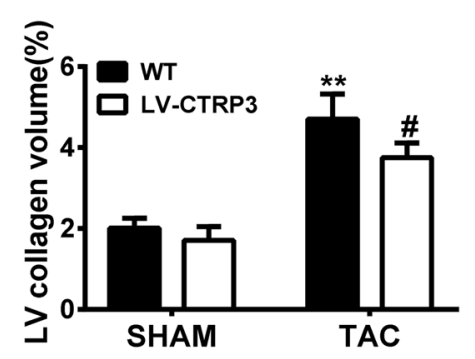

k

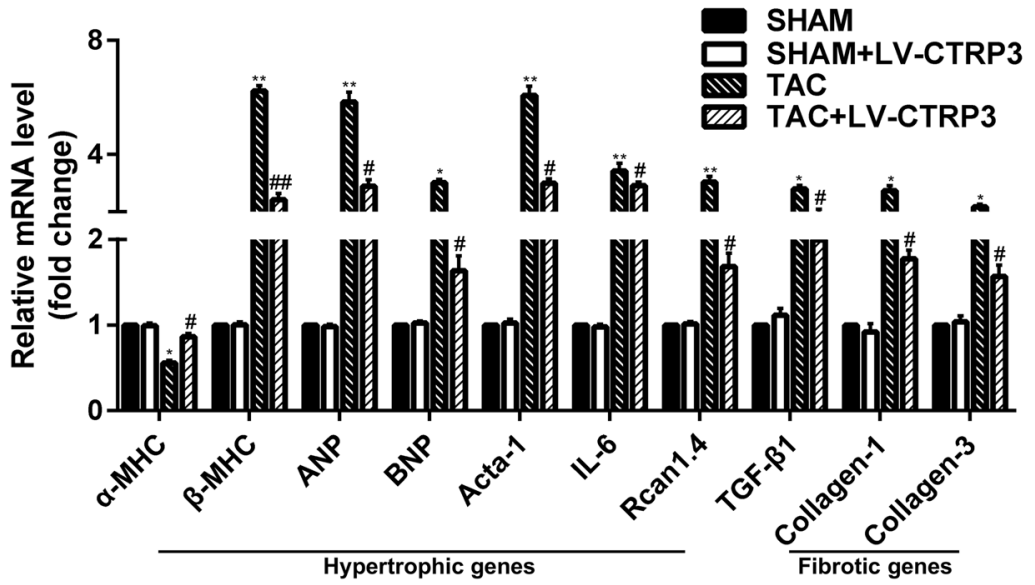

Fig. 2 (See legend on next page.) 
(see figure on previous page)

Fig. 2 CTRP3 overexpression represses pressure overload-induced cardiac hypertrophy. a Representative western blot and quantification of CTRP3 levels in the heart tissue of WT mice and LV-CTRP3 mice ( $n=3$ mice per group). b CTRP3 serum levels in mice infected with LV-NULL or LVCTRP3 ( $n=3$ mice per group). c The HW/BW ratio in animals after 4 weeks of TAC surgery $(n=5-7$ mice per group). d-f $L$ LVEF, IVSd, and LVPWd, accordingly, determined by analyzing the echocardiographic images ( $n=12-14$ mice per group). $\mathbf{g}$ Representative images of the gross murine heart and sections stained with HE and WGA ( $n=5-7$ mice per group). $\mathbf{h}$ The mean cross-sectional area of cardiomyocytes from the indicated groups $(n \geq$ 100 cells per group). i Representative images of the murine heart sections (after 4 weeks of TAC) stained with Masson stain, arranged with the perivascular area at the top and the interstitial area at the bottom ( $n=5-7$ mice per group). $\mathbf{j}$ The LV collagen volume in different groups ( $n \geq 40$ fields per group). $\mathbf{k}$ Real-time PCR analysis for the expression of genes encoding the hypertrophic markers a-MHC, $\beta-M H C, A N P, B N P, A c t a-1, I L-6$, and Rcan 1.4, and the fibrotic markers TGF- $\beta 1$, collagen-I, and collagen-III in each group ( $n=6$ mice per group). $\mathbf{c}-\mathbf{k}$ The data were analyzed by one-way ANOVA. ${ }^{*} p<0.05,{ }^{* *} p<0.01$ vs. SHAM, ${ }^{*} p<0.05$ vs. TAC; ns, not significant. In the bar graphs, the data are presented as the mean \pm SEM

the phosphorylation and expression levels of AMPactivated protein kinase (AMPK) and Akt were not affected by changes in CTRP3 expression (Fig. S6). However, after 4 weeks of TAC, the phosphorylation of p38 MAPK and its downstream target CREB were increased, and this effect was significantly potentiated in TAC-operated Ctrp3-KO mice (Fig. 4a). The upregulation of p38 and CREB phosphorylation after TAC was significantly reduced in LV-CTRP3 mice (Fig. 4b). The immunofluorescence intensity of p-p38 was stronger in TAC-operated WT mice than in sham-operated WT mice, and CTRP3 deficiency enhanced this change (Fig. 4c). The immunofluorescence intensity of p-p38 in TAC mice was also decreased by Ctrp3 overexpression (Fig. $4 d)$.

\section{Protein p38 MAPK activation mediates give-and-take of CTRP3-regulated cardiac hypertrophy and fibrosis}

To further explore the role of CTRP3 in regulating the p38 MAPK signaling pathways in TAC-operated mice, SB203580 was used to inhibit the activation of p38 MAPK. After SB203580 treatment, EF\%, HW/BW, ventricular wall thickness, cross-sectional area, and left ventricular collagen volume were markedly improved in mice from WT + TAC + SB203580 group compared to WT + TAC + DMSO group (Fig. 5). After TAC, some LVCTRP3 mice were treated with SB203580. No significant differences in $\mathrm{EF} \%, \mathrm{HW} / \mathrm{BW}$, ventricular wall thickness, cross-sectional area, and left ventricular collagen volume were apparent in the presence or absence of SB203580 in LV-CTRP3 mice after TAC (Fig. 5). This indicated that the inhibition of p38 did not affect the antihypertrophic role of CTRP3. When Ctrp3-KO mice were treated with SB203580 after TAC, the cardiac hypertrophy (Fig. 5c, d) and fibrosis (Fig. 5c, e) aggravated by CTRP3 deficiency were significantly improved, as well as the damage of cardiac function (Fig. 5a) and increased HW/BW (Fig. 5b). The expression of hypertrophic and fibrotic marker molecules was consistent with the phenotypic observations (Fig. 5f). Moreover, p38 inhibitor treatment significantly decreased the TAC-induced activation of CREB in heart. Further aggravated CREB activation in Ctrp3-KO mice was also decreased by $\mathrm{p} 38$ inhibitor. And there was no significant difference of CREB activation between TAC + LV-CTRP3 + DMSO group and TAC + LV-CTRP3 + SB203580 group. (Fig. 5g). Collectively, these findings suggested that CTRP3 inhibited pathological cardiac hypertrophy at least by inhibiting the activation of the p38/CREB signaling cascade.

\section{CTRP3 alleviates PE-induced cardiomyocyte hypertrophy in vitro by inhibiting the p38/CREB pathway}

We next proceeded to thoroughly investigate whether the p38/CREB signaling pathway participates in the antihypertrophic activity of CTRP3 in PE-induced cardiomyocyte hypertrophy. In PE-treated NRCMs, the phosphorylation level of p38 and CREB was increased, and siCTRP3 administration exaggerated this response (Fig. 6a). However, CTRP3 supplementation inhibited the p38 and CREB activation induced by PE (Fig. 6b). Further, when p38 activity in PE-treated NRCMs was suppressed by SB203580, the PE-induced increase of cell size and expression of the hypertrophic genes were alleviated, indicating that phosphorylation of p38 was indeed involved in cardiomyocyte hypertrophy (Fig. 6c, d). In the presence of CTRP3 siRNA, the PE-induced increase of cell size and hypertrophic gene expression were exaggerated, while SB203580 blunted this effect (Fig. 6c, d). Similarly, CREB activation was inhibited by SB203580 (Fig. 6e). Finally, in the presence of CTRP3, the PEinduced increase of cell size and expression of the hypertrophic genes were alleviated, and SB203580 did not change this effect (Fig. 6f, g). CREB activation was also inhibited by CTRP3 and SB203580, but there was no significant change in the reduction of CREB expression when CTRP3 and SB203580 were provided simultaneously (Fig. 6h). Collectively, these findings indicate that CTRP3 inhibited PE-induced cardiomyocyte hypertrophy, at least partially, by inhibiting the activation of the p38/ CREB signaling cascade. 


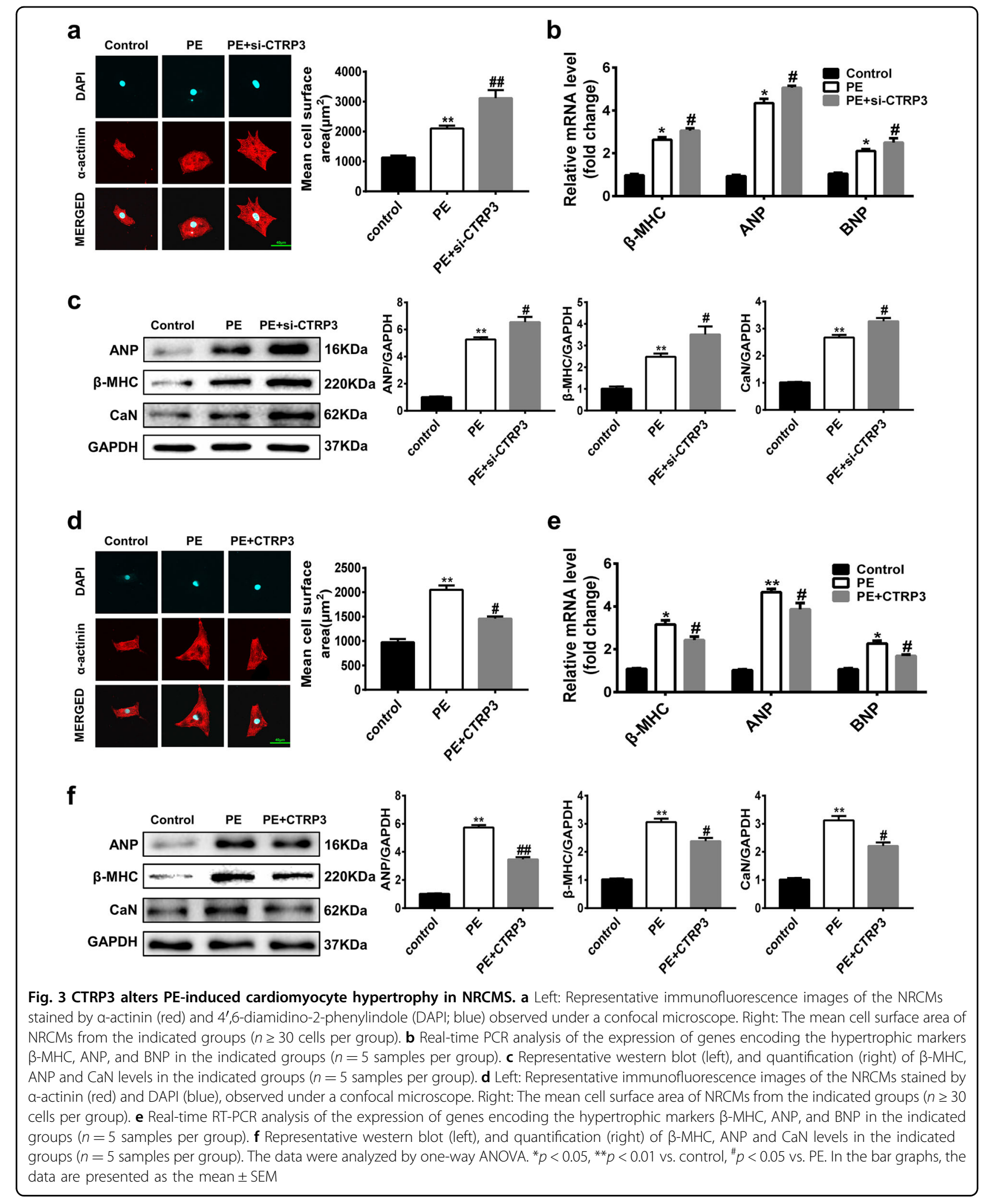



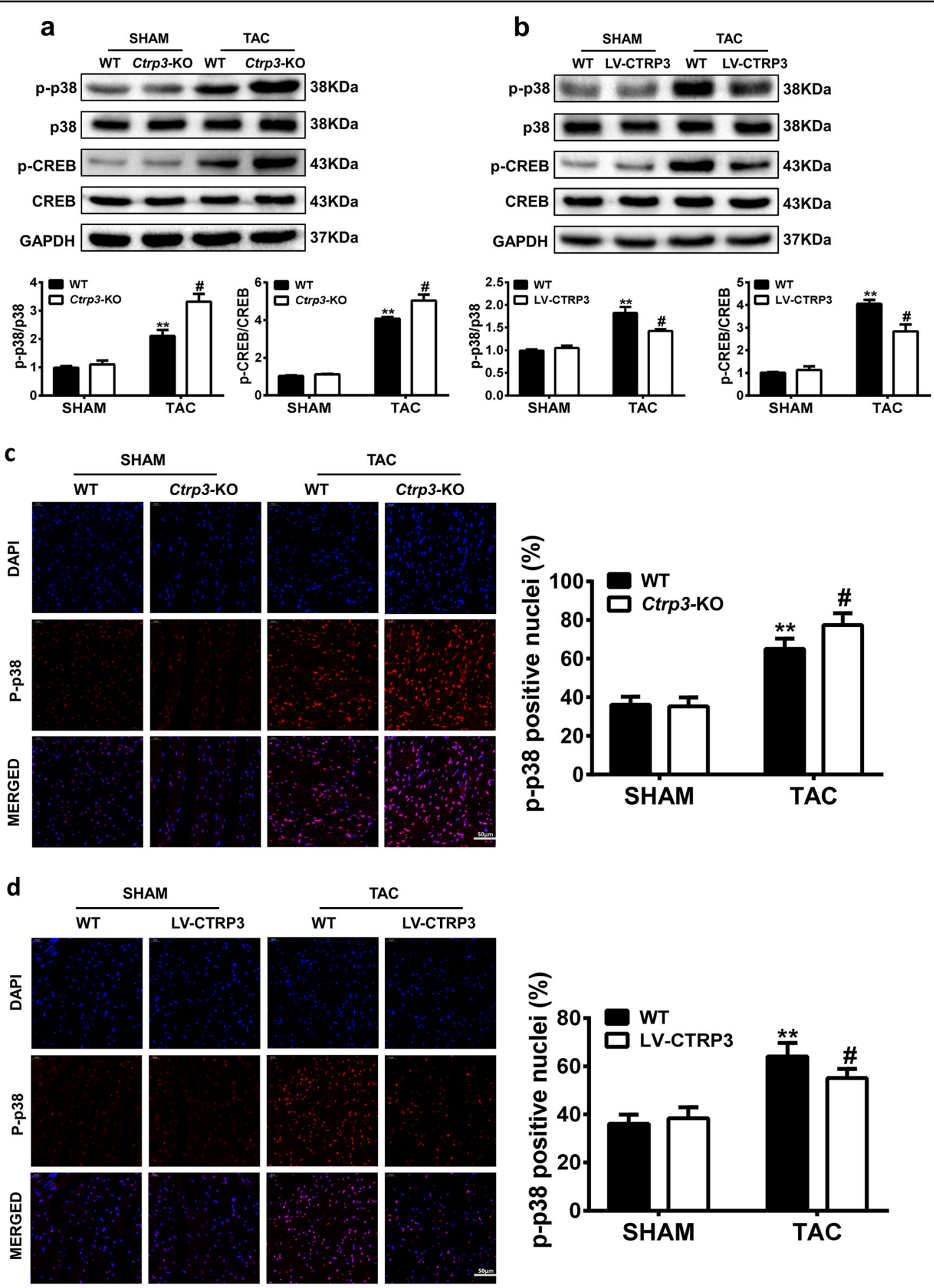

Fig. 4 CTRP3 regulates activation of the p38 MAPK/CREB pathway in TAC mouse. $\mathbf{a}, \mathbf{b}$ Representative western blot (top) and quantification (bottom) of the p38-CREB signaling pathway activity in the hearts of mice with different genotypes (WT, Ctrp3-KO, and LV-CTRP3) 4 weeks after sham treatment or TAC surgery ( $n=5-6$ mice per group). $\mathbf{c}$, $\mathbf{d}$ Representative immunofluorescence images of murine heart sections stained with $p$-p38 (red) and DAPI (blue) (left), and the percentage of p-p38-positive nuclei (right) in the hearts of mice with different genotypes (WT, Ctrp3-KO, and LVCTRP3) 4 weeks after sham treatment or TAC surgery ( $n=5-6$ mice per group). The data were analyzed by one-way ANOVA. ${ }^{*} p<0.05,{ }^{* *} p<0.01$ vs. SHAM, ${ }^{\#} p<0.05$ vs. TAC. In the bar graphs, the data are presented as the mean \pm SEM 


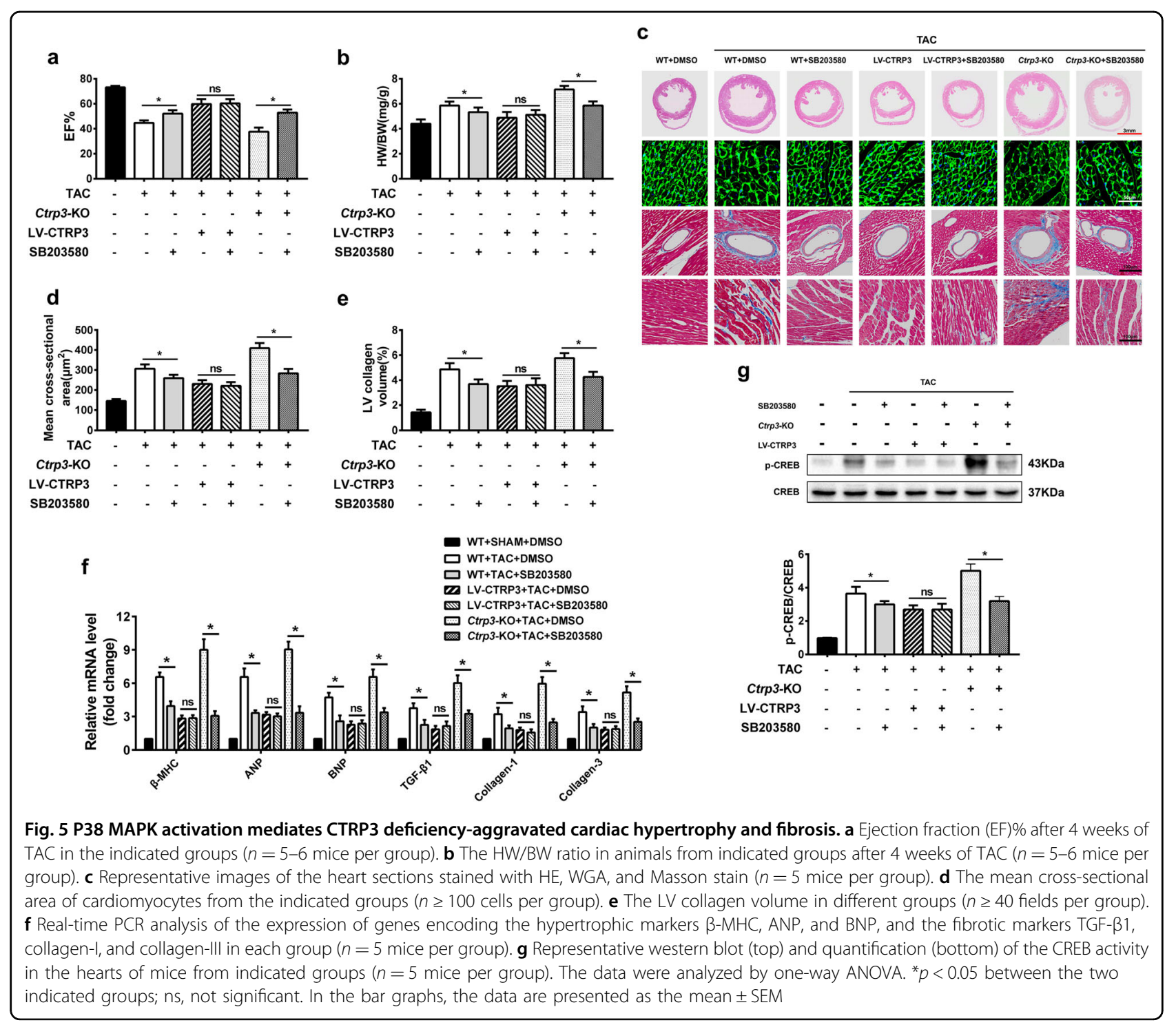

Protein p38-induced activation of the ER stress pathway is involved in pathological cardiac hypertrophy

In addition, we analyzed the protein levels of key ER stress molecules, such as GRP78 and the downstream signaling molecules eIF2 $\alpha$, CHOP, IRE1, and ATF6. CTRP3 deficiency exacerbated the TAC-induced activation of the ER stress signaling pathways (Fig. 7a-b). The immunofluorescence intensity of GRP78 was stronger in TACoperated WT mice than in sham-operated WT mice, and CTRP3 deficiency enhanced the change (Fig. 7e). CTRP3 overexpression alleviated TAC-induced activation of the ER stress signaling pathways (Fig. 7c-f). We also examined the mRNA expression level of GRP78, ATF4, ATF6, and $\mathrm{CHOP}$ in vivo and in vitro experiments to further confirm the influence of CTRP3 on the ER stress signaling pathway. The results showed that TAC-induced upregulation of ER stress signaling mRNA levels were enhanced by CTRP3 deficiency and were attenuated by CTRP3 overexpression in vivo (Fig. S7a, b). In addition, the TAC-induced ER stress and ER stress after TAC exacerbated by Ctrp3-KO were alleviated by SB203580 and there is no significant difference between WT + TAC + SB203580 group and Ctrp3-KO + $\mathrm{TAC}+\mathrm{SB} 203580$ group (Fig. 7g).

In PE-treated NRCMs, the ER stress was also activated and exaggerated in the absence of CTRP3, while CTRP3 supplementation decreased the activation of ER stress (Fig. S8). Furthermore, SB203580 blunted the activation of $E R$ stress induced by $P E$ and attenuated the exaggerated ER stress in the presence of si-CTRP3, as evidenced by analysis of GRP78, IRE, eIF $2 \alpha$, and CHOP levels. There was no significant difference on activation of ER stress between PE + siRNA + SB203580 group and PE + si-CTRP3 + SB203580 group (Fig. 8a). Next, we pretreated NRCMs with $666-15$ to inhibit activation of 

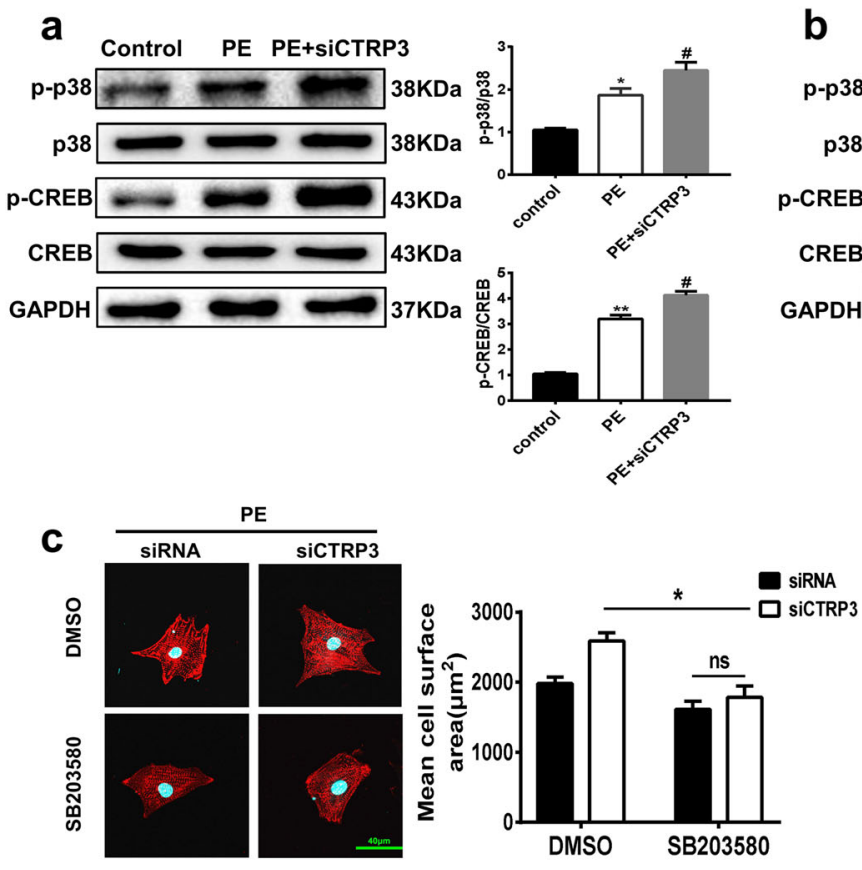

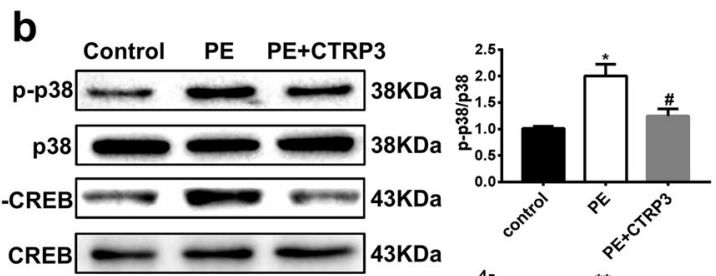

GAPDH $2-2 \mathrm{KDa}$

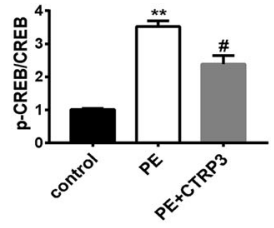

d

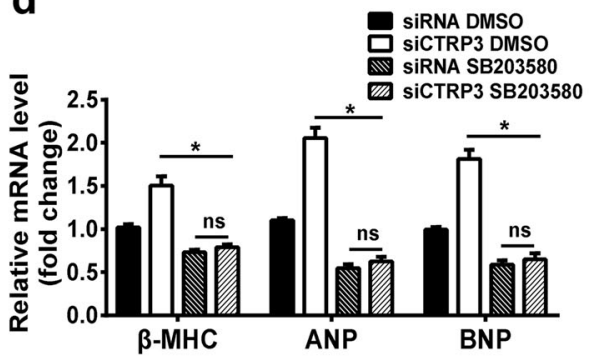

e
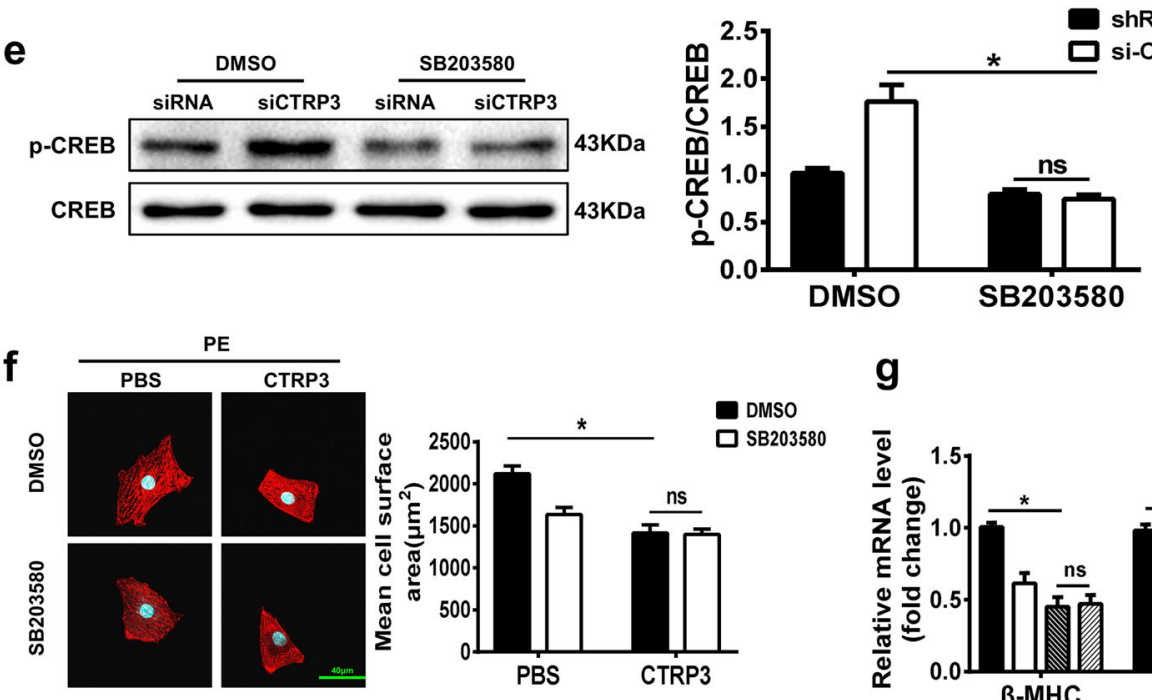

g

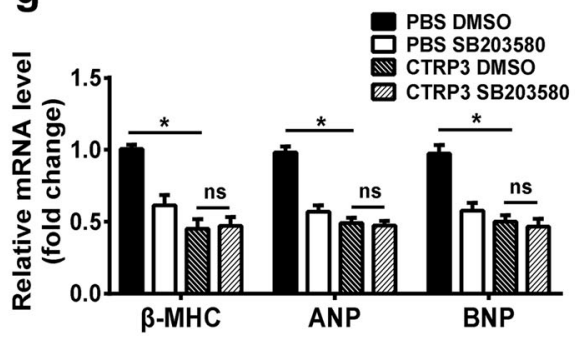

h
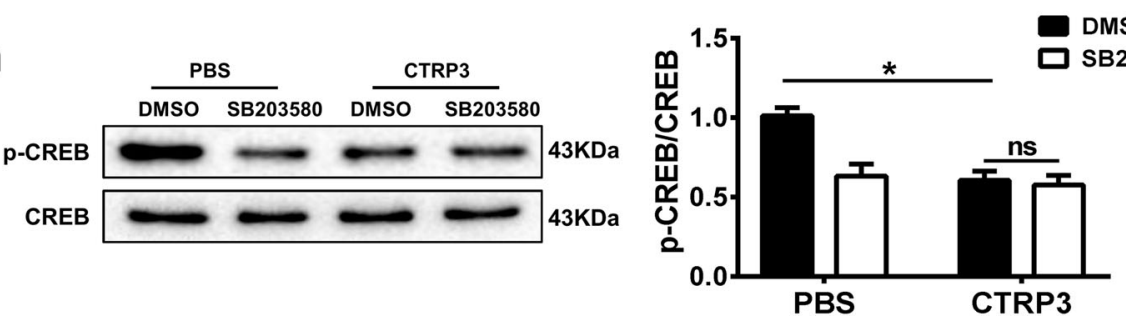

Fig. 6 (See legend on next page.) 
(see figure on previous page)

Fig. 6 CTRP3 alleviates PE-induced cardiomyocyte hypertrophy in vitro by inhibiting the p38 MAPK pathway. a Representative western blot (left) and quantification (right) of the p38-CREB signaling pathway activity in NRCMs from the indicated groups ( $n=5$ samples per group). b Representative western blot (left) and quantification (right) of the p38-CREB signaling pathway activity in NRCMs from the indicated groups ( $n=$ 5 samples per group). c Left: Representative immunofluorescence images of NRCMs transfected with siRNA or si-CTRP3, and stained with a-actinin (red) and DAPI (blue). The NRCMs were treated with dimethyl sulfoxide (DMSO) or a p38 inhibitor SB203580 (1 $\mu$ M). Right: The mean cell surface area of NRCMs in the indicated groups ( $n \geq 30$ cells per group). $\mathbf{d}$ Real-time PCR analysis of the expression of genes encoding the hypertrophic markers $\beta-M H C, A N P$, and BNP in the indicated groups ( $n=5$ samples per group). e Representative western blot (left), and quantification (right) of $p-C R E B$ and CREB levels in NRCMs from the indicated groups ( $n=5$ samples per group). $\mathbf{f}$ Left: Representative immunofluorescence images of PBS- and CTRP3treated NRCMs stained with a-actinin (red) and DAPI (blue). The NRCMs were treated with DMSO or SB203580 (1 $\mu$ M). Right: The mean cell surface area of NRCMs in the indicated groups ( $n \geq 30$ cells per group). $\mathbf{g}$ Real-time PCR analysis of the expression of genes encoding the hypertrophic markers $\beta-M H C$, ANP, and BNP in the indicated groups ( $n=4$ samples per group). $\mathbf{h}$ Representative western blot (left), and quantification (right) of $p-$ CREB and CREB levels in NRCMs from the indicated groups ( $n=5$ samples per group). The data were analyzed by one-way ANOVA. $\mathbf{a}$, and $\mathbf{b}{ }^{*} p<0.05$, ${ }^{* *} p<0.01$ vs. control, ${ }^{*} p<0.05$ vs. PE. $\mathbf{e}, \mathbf{h}{ }^{*} p<0.05$ between the two indicated groups; ns, not significant. In the bar graphs, the data are presented as the mean \pm SEM

CREB. The results showed that CREB phosphorylation was effectively inhibited by $666-15$. However, 666-15 made no obvious changes on PE-induced activation of p38. Moreover, 666-15 significantly decreased the protein expression of ANP and $\beta-\mathrm{MHC}$ and inhibited the activation of ER stress, including the expression of GRP78, CHOP, ATF6, and the phosphorylation of IRE1 in PE + siRNA + 666-15 group and PE + si-CTRP3 + 666-15 group. There was also no difference between the two groups (Fig. 8b). Finally, we treated NRCMs with 4-PBA to inhibit ER stress. We detected protein expression of GRP78, CHOP, and ATF6 and activation of IRE1 to confirm that PE-induced ER stress was inhibited. However, there is no significant change in activation levels of p38 and CREB. Furthermore, when p38 and CREB were activated to the same degree, inhibition of ER stress significantly reduced the protein expression of ANP and $\beta$-MHC in NRCMs from PE + siRNA + 4-PBA group and $\mathrm{PE}+\mathrm{si}-\mathrm{CTRP} 3+4$-PBA group. Moreover, there was no significant difference between the two groups (Fig. 8c). All these data indicated that ER stress acted as a downstream signaling pathway of $\mathrm{p} 38 / \mathrm{CREB}$ in aggravated cardiac hypertrophy induced by CTRP3 deficiency.

Combined, these observations indicated that the suppression of p38-CREB and ER stress pathways involved in the protective effects of CTRP3 against pathological cardiac hypertrophy (Fig. 8d).

\section{Discussion}

Since the 2017 ACC/AHA guidelines lowered the minimum level of hypertension to $130 / 80 \mathrm{mmHg}$, the number of adults with hypertension around the world further increased. Chronic hypertension caused long-term left ventricular pressure overload, leading to pathological cardiac hypertrophy and eventually heart failure ${ }^{29}$. It is wildly accepted that cardiac hypertrophy could act as an important therapeutic target for chronic heart failure ${ }^{5}$. But because the signaling pathway of cardiac hypertrophy is complex and imperfect, more basic and clinical studies are needed to explore the intervention strategies of cardiac hypertrophy ${ }^{30,31}$. In this study, we observed that CTRP3 deficiency aggravated the pressure overloadinduced cardiac hypertrophy and fibrosis, while CTRP3 overexpression exerted a protective effect. We also found that CTRP3 exerted these effects by inhibiting the activation of $\mathrm{p} 38-\mathrm{CREB}$ and alleviating ER stress. The antihypertrophic effect of CTRP3 was verified in the in vitro PE-induced hypertrophic model.

Firstly, TAC surgery directly constricts the internal diameter of the aortic arch and increases the afterload of the left ventricle, and is widely used to research pressureoverload pathological cardiac hypertrophy and heart failure $^{32}$. In this study, 4 weeks after TAC surgery, the heart function was weakened and myocardial fibrotic reconstruction formed. The impaired cardiac function and cardiac remodeling were deteriorated in Ctrp3-KO mice. However, these changes were attenuated by CTRP3 overexpression. After TAC, the expression of hypertrophic and fibrotic genes was significantly upregulated; CTRP3 deficiency enhanced this effect but CTRP3 overexpression mitigated it. The expression of $\alpha-\mathrm{MHC}$ decreased and the expression of $\beta$-MHC increased in mice hearts after TAC. This conversion trend became accelerated in Ctrp3-KO mice but slowed down in LV-CTRP3 mice. A prolonged or severe pressure overload in the heart causes adaptive transformation of $\alpha-\mathrm{MHC}$ to $\beta-\mathrm{MHC}^{33}$. During heart failure, the expression level of $\alpha$ MHC gene decreases in ventricular myocytes ${ }^{34}$. Studies have confirmed that a continuous high expression of $\alpha$ MHC exerts a certain protective effect on cardiac function $^{35}$. The two major downstream regulatory proteins, $\mathrm{CaN}$ and phosphorylated-CaMKII, are closely associated with the development of myocardial hypertrophy ${ }^{36}$. Activation of $\mathrm{CaN}$ can regulate the expression of genes for hypertrophy-related molecules ${ }^{37}$. Inhibition of CaMKII activity can prevent cardiac hypertrophy ${ }^{38,39}$. In this study, changes in the $\mathrm{CaN}$ and p-CaMKII protein levels matched those of changes of other hypertrophic genes. 


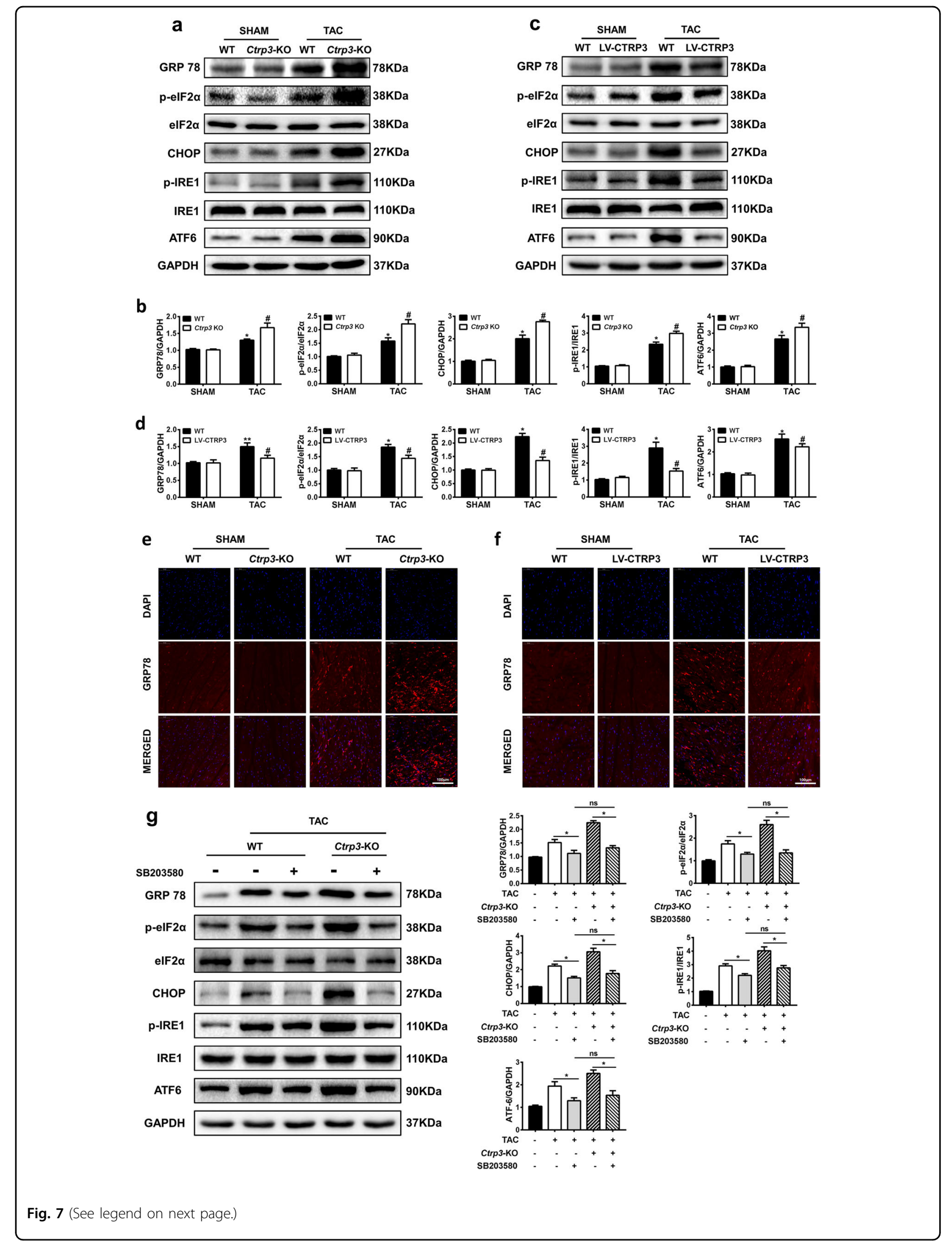


(see figure on previous page)

Fig. 7 Protein p38-induced activation of the ER stress pathway is involved in pathological cardiac hypertrophy. a Representative western blot of the activity of GRP78, elF2a, IRE1a, CHOP, and ATF6 in mice hearts from indicated groups. b Statistical diagrams of GRP78, elF2a, IRE1a, CHOP, and ATF6 protein expression in mice hearts from indicated groups ( $n=6$ mice per group). c Representative western blot of the activity of GRP78, elF2a, IRE1a, CHOP, and ATF6 in mice hearts from indicated groups. d Statistical diagrams of GRP78, elF2a, IRE1a, CHOP, and ATF6 protein expression in mice hearts from indicated groups ( $n=6$ mice per group). $\mathbf{e}, \mathbf{f}$ Representative immunofluorescence images of murine heart sections stained with GRP78 (red) and DAPI (blue) in the hearts of mice with different genotypes (WT, Ctrp3-KO, and LV-CTRP3) 4 weeks after sham treatment or TAC surgery ( $n=5-6$ mice per group). g Representative western blot (left), and quantification (right) of GRP78, elF2a, IRE1a, CHOP, and ATF6 in the hearts of mice from the indicated groups ( $n=5$ mice per group). The data were analyzed by one-way ANOVA. $\mathbf{b}, \mathbf{d}{ }^{*} p<0.05,{ }^{* *} p<0.01$ vs. SHAM, ${ }^{\#} p<0.05$ vs. TAC. $\mathbf{g}^{*} p<0.05$ and ${ }^{*} p<0.05$ between indicated groups. In the bar graphs, the data are presented as the mean \pm SEM
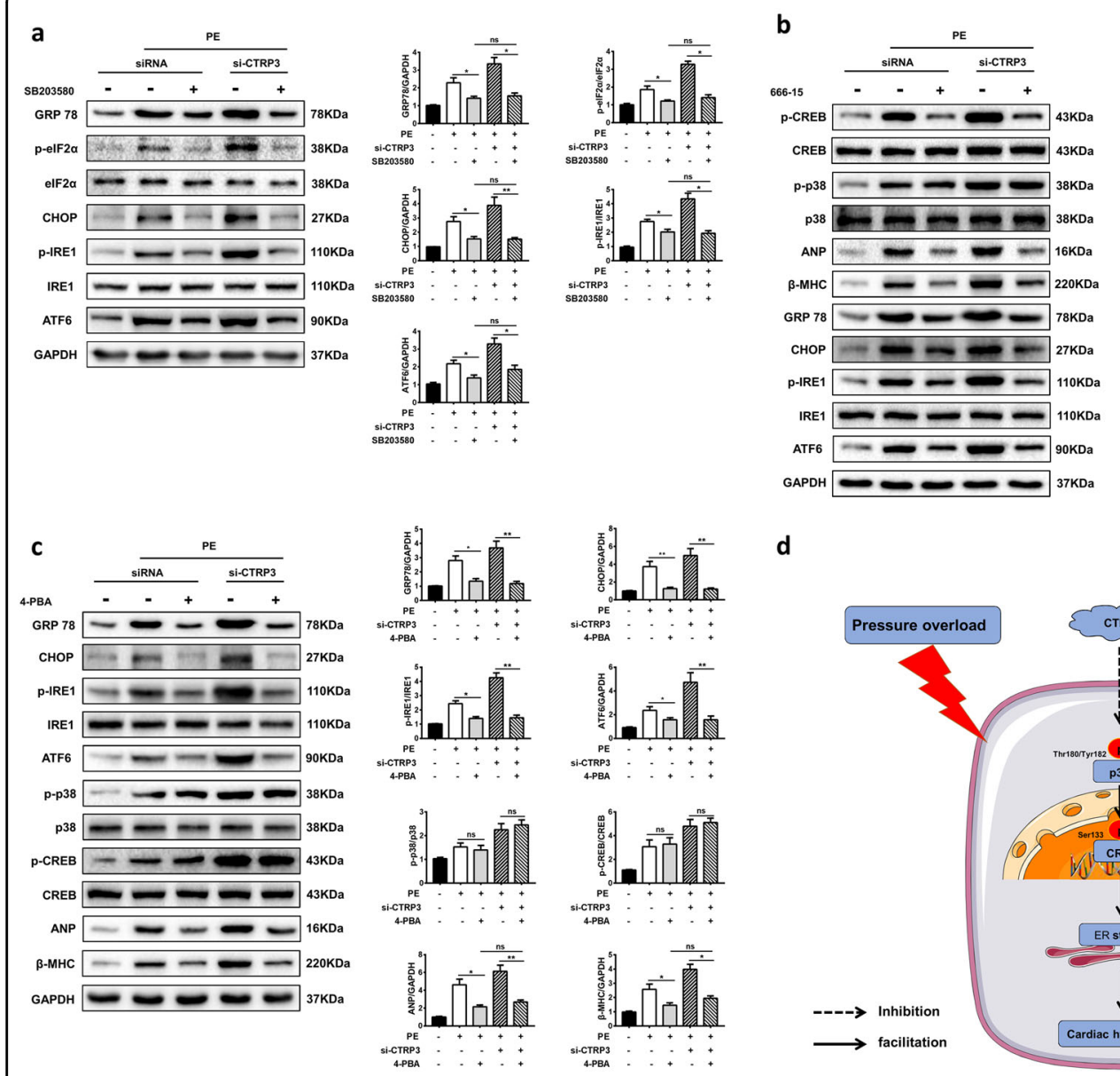
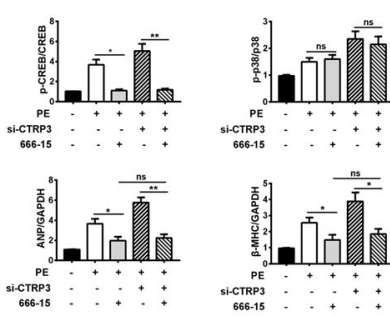

d
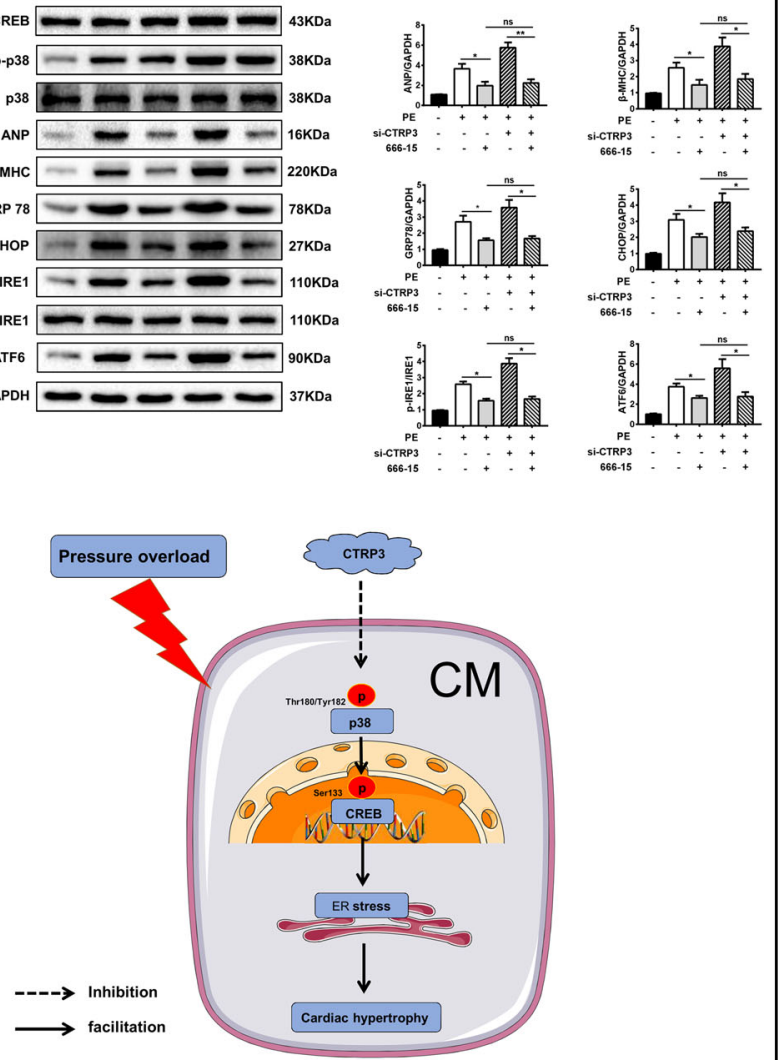

Fig. 8 CTRP3 exerted its protective role on pathological cardiac hypertrophy through inhibiting p38/CREB/ER stress pathway. a

Representative western blot (left) and quantification (right) of the ER stress signaling pathway activity in NRCMs from the indicated groups ( $n=$ 4 samples per group). b Representative western blot (left) and quantification (right) of the p38-CREB and ER stress signaling pathway activity in NRCMs from the indicated groups ( $n=4$ samples per group). c Representative western blot (left) and quantification (right) of ER stress and p38-CREB signaling pathway activity, protein expression levels of ANP and $\beta-M H C$ in NRCMs from the indicated groups ( $n=4$ samples per group). ${ }^{*} p<0.05$, ${ }^{* *} p<0.01$ between indicated groups. In the bar graphs, the data are presented as the mean \pm SEM. $\mathbf{d}$ Proposed protective mechanism of the role of CTRP3 in pathological cardiac hypertrophy. CTRP3 inhibits the activation of the p38/CREB signaling pathway and ER stress pathway in cardiomyocytes under pressure overload, thereby alleviating pathological cardiac hypertrophy

We also used PE to induce cardiac hypertrophy in $\mathrm{NCRMs}^{36}$, and the results were consistent with those of in vivo experiments. Therefore, our results suggested an improved prognosis after TAC by CTRP3 overexpression. 
Secondly, we revealed the role of the p38 MAPK/CREB signaling pathway in the anticardiac hypertrophy of CTRP3. In this study, the p-p38 and p-CREB were significantly upregulated in mice hearts after TAC, CTRP3 deficiency obviously exacerbated this effect, while CTRP3 overexpression suppressed it. Furthermore, the p38/CREB signaling pathway was confirmed to mediate give-andtake of CTRP3-regulated cardiac hypertrophy induced by TAC in vivo and PE in vitro. P38 MAPK is a stressactivated Ser/Thr kinase, activated by many pathological cardiovascular stressors, including hypertrophic stimuli ${ }^{40}$. Sustained activation of p38 contributes to myocyte hypertrophy and death in neonatal cardiomyocytes ${ }^{41,42}$. Further, multiple drugs and proteins protect against the pathological cardiac hypertrophy by inhibiting the p38 MAPK pathway ${ }^{11,43}$. CREB is a downstream effector of p38 MAPK $^{10,44}$. The phosphorylated form of CREB reduces the level of $\mathrm{Bcl}-2$ and enhances the level of the cfos, mediating the occurrence of cardiac hypertrophy ${ }^{45}$. Several molecules can attenuate cardiomyocyte hypertrophy by inhibiting phosphorylation of CREB ${ }^{16,46}$.

Thirdly, we determined the role of p38-induced ER stress in the anticardiac hypertrophy of CTRP3. TACinduced activation of ER stress including the activation of GRP78 and downstream signaling pathways, which was intensified in Ctrp3-KO mice but attenuated in LVCTRP3 mice. In vitro experiments, CTRP3 knockdown aggravated ER stress induced by $\mathrm{PE}$, while CTRP3 supplement significantly reduced it. In vitro studies proved that CTRP3 knockdown further activated p38, and enhanced ER stress in cardiomyocytes by activating downstream CREB, further aggravating the hypertrophy response of cardiomyocytes induced by PE. ER stress can be triggered by many pathological stimuli, including oxidative stress, ischemic insult, and disturbance in calcium homeostasis $^{18}$. Recently, the involvement of ER stress in the many facets of cardiovascular disease was shown, including cardiac hypertrophy ${ }^{19}$. When unfolded proteins accumulate in the ER, GRP78 breaks the inactive state of three ER transmembrane sensors (PERK, IRE1, and ATF6) to reduce the unfolded protein level ${ }^{47}$. An obvious activation of the unfolded protein response is apparent both in heart failure patients and in TAC-operated mouse $^{20,48,49}$. In TAC-operated mouse, CHOP plays a vital role in the pathological transition from cardiac hypertrophy to heart failure ${ }^{20}$. Furthermore, CHOP deficiency alleviates the cardiac hypertrophy in a mouse subjected to $\mathrm{TAC}^{50}$. All these studies suggest that ER stress is a critical molecular mechanism involved in cardiac hypertrophy. More importantly, p38 MAPK deficiency attenuates the activation of $\mathrm{CHOP}$ and cell apoptosis in a pressure-overloaded heart ${ }^{51}$. Further, in aging-induced cardiac malfunction, activated p38 MAPK mediates the activation of ER stress and cell apoptosis ${ }^{52}$, suggesting the regulating effect of p38 on ER stress. Our results confirmed that CTRP3 exerted a protective role in pathological cardiac hypertrophy at least through inhibiting the p38/CREB signaling pathway and alleviating the downstream ER stress. However, in the process of pathological cardiac hypertrophy, the direct molecule of CTRP3 in cardiac myocytes has not been clearly defined, and the downstream mechanism still needs to be further studied. Whether CTRP3 only delays the heart failure process, or can reverse pathological cardiac hypertrophy, further research is also needed.

According to our current study, we can explore a drug or agonist to intervene in patients with chronic hypertension, to a certain extent, activating the high expression of CTRP3 in myocardial tissue. CTRP3 overexpression can play a protective role in myocardium and delay the transition from cardiac hypertrophy to heart failure. Since CTRP3 supplement can also protect against PE-induced cardiomyocyte hypertrophy, direct exogenous supplementation of CTRP3 may also become an effective clinical therapeutic means, suggesting a broader clinical prospect.

Recently a study by Ma et al. showed different results that CTRP3 exacerbated cardiac hypertrophy in mice ${ }^{31}$. There are noteworthy differences about methods to abolish expression and overexpression of CTRP3 which may lead to different phenotypes. These results indicated that CTRP3 might exert different influences and mechanisms under different condition. Role and mechanism of CTRP3 in pathologic myocardial hypertrophy deserve our further studies.

In conclusion, CTRP3 protects the host against the TAC-induced cardiac hypertrophy and heart dysfunction by inhibiting the p38/CREB pathway and downstream ER stress. In vitro experiments similarly indicated that the stress-resistance role of CTRP3 in PE-induced cardiac hypertrophy was mediated by inhibiting the p38/CREB/ ER stress pathway. These results provide new perspectives for the use of CTRP3 as a therapeutic target for treating pressure-overload cardiac hypertrophy and for preventing the transition from hypertrophy to heart failure. Further studies in thorough mechanisms involved are imperative before testing CTRP3 in clinical trials.

\section{Methods \\ Experimental animals}

All procedures involving the use and care of animals were performed according to the Guide for the Care and Use of Laboratory Animals of the Chinese Animal Welfare Committee and with the approval from the Fourth Military Medical University Committee on Animal Care. Ctrp3-KO mice were constructed by eliminating the second exon in Ctrp3 gene (GenBank Accession No. 
NC_000081) using the clustered regularly interspaced short palindromic repeats (CRISPR)/Cas9 system at the Nanjing Biomedical Research Institute of Nanjing University (NBRI; Nanjing, China). Ctrp3-KO male mice and WT male C57BL/6 mice (20-25 g, 8-10-week-old, obtained from the Experimental Animal Center of the Fourth Military Medical University, Xi'an, Shaanxi, China), were housed in cages (10-12 animals/cage) under a 12:12-h light/dark cycle (lights on $06: 00$ ) at $22-24{ }^{\circ} \mathrm{C}$, and had access to a regular pellet diet ad libitum. The surgery and subsequent analyses were performed as specified below.

\section{Preparation and intramyocardial injection of the lentivirus (LV)-CTRP3 construct}

Recombinant lentivirus overexpressing mouse Ctrp3 (NM_001204134) was constructed by Shanghai Genechem Co., Ltd (Shanghai, China). WT male C57BL/6 mice (6-8 weeks old) were anesthetized in an induction chamber with $2 \%(\mathrm{v} / \mathrm{v})$ isoflurane mixed with pure oxygen $(0.5-1.0 \mathrm{l} / \mathrm{min})$. Intramyocardial injection was performed as follows ${ }^{53}$. An oblique incision $\sim 0.5-\mathrm{cm}$ long was made between the fourth and fifth costal margin of the left side of the sternum. The heart was then squeezed by exerting pressure on the right chest wall. The lentivirus was then provided by an intramuscular injection from the front, side, and back of the left ventricle, the total amount is $25 \mu \mathrm{l}\left(5 \times 10^{6}\right.$ drips of CTRP3-overexpressing lentivirus). LV-NULL was injected in a similar manner into another group of mice. The heart was then repositioned in the chest cavity and the chest wall sutured. Sham and TAC surgeries were performed 2 weeks later.

\section{Expression and production of CTRP3}

CTRP3 was overproduced and purified as previously reported $^{22}$. Briefly, the Ctrp3 gene (GenBank Accession No. NM_030945.3) was synthesized by GENEWIZ (Jiangsu, China) and cloned into the prokaryotic protein expression vector pET30a (Novagen, Merck Eurolab, Fontenay-sous-Bois, France). The expression vector was then transferred into Escherichia coli BL21(DE3) for protein production. BL21(DE3)/pET30a-CTRP3 were grown in $\mathrm{LB}$ medium $(\mathrm{NaCl} 10 \mathrm{~g} / \mathrm{L}$, tryptone $10 \mathrm{~g} / \mathrm{l}$, yeast extract $5 \mathrm{~g} / \mathrm{l}$ ), with shaking at $37^{\circ} \mathrm{C}$ for $3 \mathrm{~h}$. Isopropyl- $\beta$-Dthiogalactoside was then added to the medium (final concentration, $1 \mathrm{mM}$ ). The solution was shaken overnight at $16^{\circ} \mathrm{C}$ and then centrifuged at $5000 \times g$. Proteins were purified under native conditions on a HisTrap HP column (GE Healthcare, Madison, Wisconsin, USA), as per the manufacturer's instructions. Endotoxin was removed by passing through an endotoxin-removal column (highcapacity endotoxin removal spin column, Pierce, Thermo Fisher Scientific, San Jose, California, USA). The purified protein was then desalted and concentrated by centrifugation using Amicon ultra-15 centrifugal filter units (UFC900396, Millipore, Billerica, MA, USA).

\section{TAC}

The murine chronic cardiac hypertrophy model was established by TAC surgery. TAC surgery was performed as previously described ${ }^{54}$. Briefly, C57BL/6 mice were anesthetized in an induction chamber with $2 \%$ isoflurane mixed with pure oxygen $(0.5-1.0 \mathrm{l} / \mathrm{min})$. The animals were orally intubated with a 20-gauge tube and ventilated (Minivent Type 845, Hugo Sachs Electronic, March, Germany) at $100-120$ times per minute $(0.15-\mathrm{ml}$ tidal volume). A median thoracotomy was performed at the second intercostal space. The transverse aorta was constricted by using a 7-0 silk suture ligature tied firmly against a 27-gauge needle between the carotid arteries. The needle was then promptly removed. Sham-operated mice underwent an identical surgical procedure except for the ligation of the aorta. The chest was closed with a 6-0 silk suture and the skin was closed with a 5-0 silk suture. After TAC, the mice were kept warm on a $38^{\circ} \mathrm{C}$ constant temperature plate and carefully monitored until free to move.

\section{Experimental design and treatment}

Fifteen WT mice were randomly selected as the SHAM group. Twelve WT mice and twelve Ctrp3-KO mice were randomly selected as the SHAM + LV-CTRP3 group and SHAM + CTRP3-KO group, respectively. Fifteen SHAM mice were selected for intramyocardial injection of LVNULL, and twelve SHAM + LV-CTRP3 mice were given intramyocardial injection of LV-CTRP3. Two weeks later, all the above mice underwent sham surgery.

Fifteen WT mice were randomly selected as the TAC group. Twelve WT mice and twelve Ctrp3-KO mice were randomly selected as the TAC + LV-CTRP3 group and TAC + CTRP3-KO group, respectively. Fifteen TAC mice were selected for intramyocardial injection of LV-NULL, and twelve TAC + LV-CTRP3 mice were given intramyocardial injection of LV-CTRP3. Two weeks later, all the above mice underwent TAC surgery.

Thirty WT mice were randomly selected and separately divided into TAC + SB203580, TAC + LV-CTRP3, and $\mathrm{TAC}+\mathrm{LV}-\mathrm{CTRP} 3+\mathrm{SB} 203580$ groups (10 mice/group). Twenty Ctrp3-KO mice were randomly selected, and separately arranged into TAC + CTRP3-KO group and $\mathrm{TAC}+\mathrm{CTRP} 3-\mathrm{KO}+\mathrm{SB} 203580$ groups (10 mice/group). SB203580 (a p38 MAPK inhibitor, purchased from Calbiochem (La Jolla, CA, USA)) was injected intraperitoneally into mice from the indicated groups every day after TAC operation. To do this, SB203580 was dissolved in 1\% DMSO $(1 \mathrm{mg} / \mathrm{ml})$. The mice were then injected intraperitoneally with SB203580 $(10 \mathrm{mg} / \mathrm{kg})^{55}$. The remaining mice were injected with the same amount of $1 \%$ DMSO. 


\section{NRCM culture}

NRCMs were obtained from the ventricles of newly born Sprague Dawley (SD) rats (male, obtained from the Experimental Animal Center of the Fourth Military Medical University, Xi'an, Shaanxi, China), as described previously $^{56}$. Briefly, newly born SD rats were disinfected twice with $75 \%$ alcohol, the heart was harvest and then cut up in serum-free Dulbecco's Modified Eagle's Medium/ Nutrient Mixture F-12 Ham (DME/F-12, 1:1 mixture; Gibco, Carlsbad, CA, USA). Then, the tissue fragments were digested in PBS solution containing 1\% collagenase-I (Sigma V900891; Sigma-Aldrich, St. Louis, MO, USA), three times, $5 \mathrm{~min}$ each time. Cell suspension was then placed in DME/F-12 containing 10\% serum, centrifuged for $5 \mathrm{~min}$ at $1000 \mathrm{rpm}$, and the supernatant was discarded. The cells were resuspended in the culture medium containing serum [DME/F-12, 10\% new bovine serum (Gibco, Carlsbad, CA, USA), penicillin $(100 \mathrm{U} / \mathrm{ml})$, streptomycin $(100 \mathrm{U} / \mathrm{ml})$, and bromodeoxyuridine (BrdU) $(0.1 \mathrm{mM}$; to inhibit fibroblast proliferation)], and placed in a culture box for $1-1.5 \mathrm{~h}$, using the differential velocity adherent method to purify the myocardial cells. The myocytes were plated in 6-well plates or confocal dishes at a density of $5 \times 10^{5}$ cells $/ \mathrm{ml}$ and cultured for $24 \mathrm{~h}$ at $37^{\circ} \mathrm{C}$ with $5 \%$ $\mathrm{CO}_{2}$. The NRCMs were then incubated with PE $(50 \mu \mathrm{M})$ for $24 \mathrm{~h}$ to induce cardiomyocyte hypertrophy. Successful induction of the cardiomyocytes was determined by measuring the cell surface area (after $\alpha$-actinin staining) and hypertrophy-related gene expression levels ( $\beta$-MHC and ANP). A part of NRCMs were firstly transfected with si-CTRP3 using Lipofectamine $2000^{27}$ (Thermo Fisher Scientific, San Jose, California, USA) before PE treatment. Some NRCMs were also treated with CTRP3 $(5 \mu \mathrm{g} / \mathrm{ml})^{22}$ in the presence of PE for $24 \mathrm{~h}$. Before PE treatment, some NRCMs were pretreated respectively with $1 \mu \mathrm{M}$ SB203580 or 1 $1 \mathrm{M}$ 666-15 (CREB inhibitor, purchased from Med Chem Express) for $2 \mathrm{~h}$ to inhibit phosphorylation of $\mathrm{p} 38$ or $\mathrm{CREB}^{57}$. In order to inhibit ER stress, some NRCMs were treated with $2 \mathrm{mM}$ 4-PBA (ER stress inhibitor, purchased from Sigma-Aldrich, St Louis, MO, USA) in the presence or absence of PE for $24 \mathrm{~h}^{58}$. The NRCMs after PE treatment were subsequently prepared for use in other experiments as described below.

\section{Echocardiography}

The echocardiographer was blinded to the experimental protocols and surgical procedure. Before measuring the echocardiography, mouse was anesthetized in an induction chamber with $2 \%$ isoflurane mixed with pure oxygen $(0.5-1.01 / \mathrm{min})$. The thermostatic plate was adjusted to $42^{\circ} \mathrm{C}$ and the mouse body temperature was maintained at about $38^{\circ} \mathrm{C}$. The heart rate of the mouse was maintained at $400-500$ beats per minute by adjusting the concentration of isoflurane. Transthoracic ultrasonography was performed using a VisualSonics 770 echocardiograph (VisualSonics 770, Toronto, ON, Canada), and a $30-\mathrm{MHz}$ transducer was used to record the views in both parasternal long-axis and short-axis of the left ventricle. LVEF was used to represent the left ventricular contractile function. IVSd and LVPWd were determined as indicators of cardiac hypertrophy. The above parameters were measured and calculated by Vevo Lab 3.1.0 software (FUJIFILM VisualSonics, Inc. Toronto, ON, Canada).

\section{Histological analysis}

Four weeks after the sham or TAC surgery, 5-6 mice from each group were weighed and then euthanized by carotid artery bleeding under anesthesia. The heart was exposed by shear, the aorta was occluded, and incision was made in the inferior vena cava. The heart was perfused by $1 \mathrm{ml}$ syringe with phosphate-buffered saline (PBS) to flush the blood out of the heart via the cardiac apex, twice, and was then perfused with $4 \%$ paraformaldehyde. The heart was then harvested and fixed in $4 \%$ paraformaldehyde in a $1.5-\mathrm{ml}$ tube at $4{ }^{\circ} \mathrm{C}$ for at least $24 \mathrm{~h}$. It was cut into 5 - $\mu \mathrm{m}$-thick sections from the cardiac apex, and the sections were stained with HE, WGA, and Masson trichrome stain to evaluate the cross-sectional area of the cardiomyocytes and the degree of fibrosis. The sections were visualized by a digital scanning imaging system Olympus FV1000 (Olympus, Tokyo, Japan) and the cross-sectional area of the cardiomyocytes and degree of fibrosis were quantified by using Image $\mathrm{J}$ software (NIH, Bethesda, MD, USA).

\section{Quantitative real-time PCR}

Mice from each group other than the ones used for histological analysis were weighed and then euthanized as mentioned above. The hearts were harvested and washed with PBS; HW was then recorded. The HW/BW ratio was then calculated. A small portion was cut off from the left ventricular wall and placed in a $1.5-\mathrm{ml}$ tube containing $1 \mathrm{ml}$ Trizol reagent (TIANGEN, Beijing, China). Total RNA was extracted from the left ventricular tissue specimen (and the cultured NRCMs) according to the protocol of the total RNA extraction kit (TIANGEN, Beijing, China). The RNA was then reverse-transcribed into complementary DNA. The CFX96 real-time PCR system C1000 Thermal Cycler (Bio-Rad Laboratories, Hercules, CA, USA) was used for real-time PCR. GAPDH served as the standard gene for the normalization of transcript levels of target genes. The primers used in this study were synthesized by GenScript Biotech Corp. (Nanjing, China) and the sequences are listed in Table S1.

\section{Western blotting}

Total protein from the mouse left ventricular wall and interventricular septum, and from the treated NRCMs 
was extracted in the RIPA lysis buffer ${ }^{56}$. The protein concentration in samples was determined using the bicinchoninic acid assay (Solarbio co, LTD, Shanghai, China). Extracted protein was separated by $10 \%$ sodium dodecyl sulfate-polyacrylamide gel electrophoresis (SDSPAGE). Then, proteins from the SDS-PAGE gel were transferred onto a polyvinylidene fluoride membrane (Millipore, Billerica, MA, USA). Membranes were blocked in 5\% skim milk powder and dissolved in TBST buffer [150 mM NaCl, $50 \mathrm{mM}$ Tris (pH 7.5), and 0.1\% Tween20) for $2-3 \mathrm{~h}$ at $20-25^{\circ} \mathrm{C}$. The membranes were then cut horizontally and incubated with the respective primary antibody overnight at $4{ }^{\circ} \mathrm{C}$. The membrane strips were washed with TBST and then incubated with the secondary antibody conjugated with horseradish peroxidase for $2 \mathrm{~h}$ at $20-25^{\circ} \mathrm{C}$. The ECL reagent (Millipore, Billerica, MA, USA) was added and the blots were scanned using ChemiDoc $^{\mathrm{Tm}}$ XRS (Bio-Rad Laboratories, Hercules, CA, USA). The gray value of protein bands was determined using Image Lab 2.0(Genmall Biotechnology Co.,Ltd, Wuhan, China) and GADPH was used as the internal control. The primary antibodies used were anti-p-CREB (Ser133; 9198 s), anti-CREB (9197 s), anti-p-CaMKII (12716), antip-eIF2 $\alpha$ (3398), anti-eIF2 $\alpha$ (5324), anti-ATF4 (11815), anti-CHOP (2895), anti-p-AMPK $\alpha$ (2537), anti-AMPK $\alpha$ (5832), anti-p-AKT (4060), anti-AKT (9272), anti-Bcl-2 (3498), and anti-Bax (5023) antibodies, purchased from Cell Signaling Technology (Beverly, MA, USA); anti-ANP (ab209232), anti-CaN A (ab3673), anti-CaMK $\square$ (ab52476), anti-GRP78 (ab21685), anti-p-IRE1 (ab48187), anti-IRE1 (ab37073), anti-XBP1 (ab37152), anti-ATF6 (ab203119), and anti-CTRP3 (ab36870) antibodies, purchased from Abcam (Cambridge, MA, USA); anti-p-p38 (sc-166182), anti-p38 (sc-7149), and anti-MYH7 (sc53089) antibodies, purchased from Santa Cruz Biotechnology (Santa Cruz, CA, China); and anti-GAPDH (AT0002) antibody purchased from cmcTAG (Milwaukee, WI, USA). The secondary antibodies used were goat anti-rabbit (ZB-2301) and goat anti-mouse (ZB-2305) antibodies purchased from Zhongshan Company (Beijing, China).

\section{Immunostaining}

Immunofluorescence staining was performed as described previously ${ }^{59}$. First, the expression and activation of p38 and GRP78 were detected by immunofluorescence. Briefly, sections were permeabilized in $0.05 \%$ Triton X100 for $10 \mathrm{~min}$. They were washed three times with PBS and incubated with $1 \%$ bovine serum albumin for $1 \mathrm{~h}$ at room temperature. The sections were incubated with mouse anti-p-p38 antibody or rabbit anti-GRP78 antibody (details in western blot chapter, both 1:50 dilution) for $10-12 \mathrm{~h}$ at $4{ }^{\circ} \mathrm{C}$. Antigen-antibody complexes were visualized by goat anti-mouse or anti-rabbit IgG antibodies were conjugated with Alexa Fluor ${ }^{\circ} \mathrm{Cy} 3$. Nuclei were visualized by DAPI. The cardiomyocytes were then counted. The p-p38 MAPK activity was expressed as the average percentage of nuclei positive for p-p38 MAPK per section.

Immunofluorescence staining was also used to determine the surface area of cardiac myocytes in each animal group. After treatment, confocal dish containing NRCMs was washed with PBS, three times, and then fixed with $4 \%$ paraformaldehyde at $4{ }^{\circ} \mathrm{C}$ for $30 \mathrm{~min}$. After washing three times with PBS, the cells were permeabilized with $0.05 \%$ Triton X-100 for $10 \mathrm{~min}$, and then washed again three times with PBS. Next, they were incubated with $1 \%$ bovine serum albumin for $30 \mathrm{~min}$ at room temperature, followed by incubations with $\alpha$-actinin (Sigma A7732, SigmaAldrich, St. Louis, MO, USA) for $10-12 \mathrm{~h} 4{ }^{\circ} \mathrm{C}$. After incubating with a secondary antibody labeled with horseradish peroxidase at $37^{\circ} \mathrm{C}$ for $2 \mathrm{~h}$, the cells were washed three times with PBS. The nucleus was stained with DAPI (purchased from BIO-LIEESCICO.,LTD, Guangzhou, China) and the cells were washed three times with PBS. The cells were then observed under a confocal microscope. For each group, at least 50 cells were analyzed at random. The Image J software (NIH) was used to calculate the cardiomyocyte surface area.

\section{ELISA}

Mouse carotid blood was collected by carotid artery bleeding. After $6 \mathrm{~h}, 1 \mathrm{ml}$ of the whole blood was centrifuged at $3000 \mathrm{rpm}$ for $10 \mathrm{~min}$. The upper serum layer was aspirated into a $1.5-\mathrm{ml}$ centrifugation tube and the serum CTRP3 level was detected by ELISA (SEK169Mu, CLOUD-CLONE CORP., Wuhan, China). All sample handling was performed according to the instructions of the ELISA kit manufacturer.

\section{Statistical analysis}

All generated data were processed and analyzed using GraphPad Prism 7.0 (GraphPad Software, In., San Diego, CA, USA). In all bar graphs, the data are presented as the mean \pm SEM. The differences between two groups were analyzed by the $t$-test. Statistical differences between multiple groups were analyzed by one-way ANOVA. In this study, $p<0.05$ represents a statistically significant difference.

\footnotetext{
Acknowledgements

This work was supported by the National Natural Science Foundation of China (grant nos. 81670356, 81770345, 81470480, 81470477, and 81870266); National High-Level Talents Special Support Plan; and the National Key Research and Development Program of China (grant no. 2016YFC1301900).
}

\section{Author details}

${ }^{1}$ Department of Cardiovascular Surgery, Xijing Hospital, The Fourth Military Medical University, 127 Changle West Road, 710032 Xi'an, China. ${ }^{2}$ Department of Geriatrics, Xijing Hospital, The Fourth Military Medical University, 127 
Changle West Road, 710032 Xi'an, China. ${ }^{3}$ Center for Translational Medicine, Lewis Katz School of Medicine at Temple University, Philadelphia, PA 19140, USA

\section{Conflict of interest}

The authors declare that they have no conflict of interest.

\section{Publisher's note}

Springer Nature remains neutral with regard to jurisdictional claims in published maps and institutional affiliations.

Supplementary Information accompanies this paper at (https://doi.org/ 10.1038/s41419-019-1749-0).

Received: 5 December 2018 Revised: 25 March 2019 Accepted: 7 May 2019 Published online: 08 July 2019

\section{References}

1. Oka, T., Akazawa, H., Naito, A. T. \& Komuro, I. Angiogenesis and cardiac hypertrophy: maintenance of cardiac function and causative roles in heart failure. Circ. Res. 114, 565-571 (2014).

2. Deng, K. Q. et al. Restoration of circulating MFGE8 (Milk Fat Globule-EGF Factor 8) attenuates cardiac hypertrophy through inhibition of akt pathway. Hypertension 70, 770-779 (2017)

3. Hill, J. A. \& Olson, E. N. Cardiac plasticity. N. Engl. J. Med. 358, 1370-1380 (2008),

4. McKinsey, T. A. \& Kass, D. A. Small-molecule therapies for cardiac hypertrophy: moving beneath the cell surface. Nat. Rev. Drug Discov. 6, 617-635 (2007).

5. Schiattarella, G. G. \& Hill, J. A. Inhibition of hypertrophy is a good therapeutic strategy in ventricular pressure overload. Circulation 131, 1435-1447 (2015).

6. Tanai, E. \& Frantz, S. Pathophysiology of heart failure. Compr. Physiol. 6, 187-214 (2015).

7. $L i$, J. et al. Arctiin protects against cardiac hypertrophy through inhibiting MAPKs and AKT signaling pathways. J. Pharmacol. Sci. 135, 97-104 (2017).

8. Wei, W. Y., Ma, Z. G., Xu, S. C., Zhang, N. \& Tang, Q. Z. Pioglitazone protected against cardiac hypertrophy via inhibiting AKT/GSK3beta and MAPK signaling pathways. PPAR Res. 2016, 9174190 (2016).

9. Ren, J. et al. Caffeic acid phenethyl ester attenuates pathological cardiac hypertrophy by regulation of MEKJERK signaling pathway in vivo and vitro. Life Sci. 181, 53-61 (2017).

10. Liu, B. et al. ALDH2 protects against alcoholic cardiomyopathy through a mechanism involving the p38 MAPK/CREB pathway and local reninangiotensin system inhibition in cardiomyocytes. Int. J. Cardiol. 257, 150-159 (2018).

11. Wang, S. et al. Choline inhibits angiotensin II-induced cardiac hypertrophy by intracellular calcium signal and p38 MAPK pathway. Naunyn Schmiedeberg's Arch. Pharmacol. 385, 823-831 (2012).

12. Yang, J. et al. Inhibition of farnesyl pyrophosphate synthase attenuates angiotensin II-induced cardiac hypertrophy and fibrosis in vivo. Int. J. Biochem. Cell Biol. 45, 657-666 (2013).

13. Wang, Y. W., Chen, X., Ma, R. \& Gao, P. Understanding the CREB1-miRNA feedback loop in human malignancies. Tumour Biol. 37, 8487-8502 (2016).

14. Steven, A. \& Seliger, B. Control of CREB expression in tumors: from molecular mechanisms and signal transduction pathways to therapeutic target. Oncotarget 7, 35454-35465 (2016).

15. Li, L. et al. Angiotensin II increases periostin expression via Ras/p38 MAPK CREB and ERK1/2/TGF-beta1 pathways in cardiac fibroblasts. Cardiovasc. Res. 91, 80-89 (2011).

16. Wang, L. et al. UCR1C is a novel activator of phosphodiesterase 4 (PDE4) long isoforms and attenuates cardiomyocyte hypertrophy. Cell. Signal. 27, 908-922 (2015).

17. Doser, T. A. et al. Transgenic overexpression of aldehyde dehydrogenase-2 rescues chronic alcohol intake-induced myocardial hypertrophy and contractile dysfunction. Circulation 119, 1941-1949 (2009).

18. Minamino, T., Komuro, I. \& Kitakaze, M. Endoplasmic reticulum stress as a therapeutic target in cardiovascular disease. Circ. Res. 107, 1071-1082 (2010).

19. Minamino, T. \& Kitakaze, M. ER stress in cardiovascular disease. J. Mol. Cell. Cardiol. 48, 1105-1110 (2010).
20. Okada, K. et al. Prolonged endoplasmic reticulum stress in hypertrophic and failing heart after aortic constriction: possible contribution of endoplasmic reticulum stress to cardiac myocyte apoptosis. Circulation 110, 705-712 (2004).

21. Park, C. S., Cha, H., Kwon, E. J., Sreenivasaiah, P. K. \& Kim, D. H. The chemical chaperone 4-phenylbutyric acid attenuates pressure-overload cardiac hypertrophy by alleviating endoplasmic reticulum stress. Biochem. Biophys. Res. Commun. 421, 578-584 (2012).

22. Yi, W. et al. C1q/tumor necrosis factor-related protein-3, a newly identified adipokine, is a novel antiapoptotic, proangiogenic, and cardioprotective molecule in the ischemic mouse heart. Circulation 125, 3159-3169 (2012).

23. Sun, Y. et al. C19/tumor necrosis factor-related protein-9, a novel adipocytederived cytokine, attenuates adverse remodeling in the ischemic mouse heart via protein kinase A activation. Circulation 128, S113-S120 (2013).

24. Zheng, Q. et al. C1q/TNF-related proteins, a family of novel adipokines, induce vascular relaxation through the adiponectin receptor-1/AMPK eNOS/nitric oxide signaling pathway. Arterioscler. Thromb. Vasc. Biol. 31, 2616-2623 (2011).

25. Maeda, T. \& Wakisaka, S. CTRP3/cartducin is induced by transforming growth factor-beta1 and promotes vascular smooth muscle cell proliferation. Cell Biol. Int. 34, 261-266 (2010).

26. Hou, M., Liu, J., Liu, F., Liu, K. \& Yu, B. C1q tumor necrosis factor-related protein3 protects mesenchymal stem cells against hypoxia- and serum deprivationinduced apoptosis through the phosphoinositide 3-kinase/Akt pathway. Int. J. Mol. Med. 33, 97-104 (2014).

27. Wu, D. et al. CTRP3 attenuates post-infarct cardiac fibrosis by targeting Smad3 activation and inhibiting myofibroblast differentiation. J. Mol. Med. (Berl.) 93, 1311-1325 (2015).

28. Mu, Y., Yin, T. L., Yin, L., Hu, X. \& Yang, J. CTRP3 attenuates high-fat diet-induced male reproductive dysfunction in mice. Clin. Sci. 132, 883-899 (2018). (London, England: 1979).

29. Yu, C. J. et al. ZNF307 (Zinc Finger Protein 307) acts as a negative regulator of pressure overload-induced cardiac hypertrophy. Hypertension 69, 615-624 (2017).

30. Tham, Y. K, Bernardo, B. C., Ooi, J. Y., Weeks, K. L. \& McMullen, J. R. Pathophysiology of cardiac hypertrophy and heart failure: signaling pathways and novel therapeutic targets. Arch. Toxicol. 89, 1401-1438 (2015).

31. Ma, Z. G. et al. Long title: C1q-tumor necrosis factor-related protein-3 exacerbates cardiac hypertrophy in mice. Cardiovasc. Res. https:/doi.org/ 10.1093/cvr/cry279 (2018).

32. Rockman, H. A. et al. Segregation of atrial-specific and inducible expression of an atrial natriuretic factor transgene in an in vivo murine model of cardiac hypertrophy. Proc. Natl Acad. Sci. USA 88, 8277-8281 (1991).

33. VanBuren, P., Harris, D. E., Alpert, N. R. \& Warshaw, D. M. Cardiac V1 and V3 myosins differ in their hydrolytic and mechanical activities in vitro. Circ. Res. 77, 439-444 (1995).

34. Nakao, K., Minobe, W., Roden, R., Bristow, M. R. \& Leinwand, L. A. Myosin heavy chain gene expression in human heart failure. J. Clin. Investig. 100, 2362-2370 (1997).

35. James, J. et al. Forced expression of alpha-myosin heavy chain in the rabbit ventricle results in cardioprotection under cardiomyopathic conditions. Circulation 111, 2339-2346 (2005).

36. Pan, Z. W. et al. Scutellarin exerts its anti-hypertrophic effects via suppressing the Ca2+-mediated calcineurin and CaMKIl signaling pathways. Naunyn Schmiedeberg's Arch. Pharmacol. 381, 137-145 (2010).

37. Wikins, B. J. \& Molkentin, J. D. Calcium-calcineurin signaling in the regulation of cardiac hypertrophy. Biochem. Biophys. Res. Commun. 322, 1178-1191 (2004).

38. Kato, T. et al. Calmodulin kinases II and IV and calcineurin are involved in leukemia inhibitory factor-induced cardiac hypertrophy in rats. Circ. Res. 87, 937-945 (2000).

39. Zhu, W. et al. $\mathrm{Ca}^{2+} / \mathrm{calmodulin-dependent} \mathrm{kinase} \mathrm{II} \mathrm{and} \mathrm{calcineurin} \mathrm{play} \mathrm{critical}$ roles in endothelin-1-induced cardiomyocyte hypertrophy. J. Biol. Chem. 275, 15239-15245 (2000).

40. Marber, M. S., Rose, B. \& Wang, Y. The p38 mitogen-activated protein kinase pathway-a potential target for intervention in infarction, hypertrophy, and heart failure. J. Mol. Cell. Cardiol. 51, 485-490 (2011).

41. Zechner, D., Thuerauf, D. J., Hanford, D. S., McDonough, P. M. \& Glembotski, C. C. A role for the p38 mitogen-activated protein kinase pathway in myocardial cell growth, sarcomeric organization, and cardiac-specific gene expression. J. Cell Biol. 139, 115-127 (1997). 
42. Wang, Y. et al. Cardiac muscle cell hypertrophy and apoptosis induced by distinct members of the p38 mitogen-activated protein kinase family. J. Biol. Chem. 273, 2161-2168 (1998).

43. Chen, Y. et al. Endogenous BNP attenuates cardiomyocyte hypertrophy induced by Ang II via p38 MAPK/Smad signaling. Die Pharm. 69, 833-837 (2014).

44. Li, B., Kaetzel, M. A. \& Dedman, J. R. Signaling pathways regulating murine cardiac CREB phosphorylation. Biochem. Biophys. Res. Commun. 350, 179-184 (2006).

45. Fladmark, K. E. et al. $\mathrm{Ca}^{2+} /$ calmodulin-dependent protein kinase $\|$ is required for microcystin-induced apoptosis. J. Biol. Chem. 277, 2804-2811 (2002).

46. Tang, $X$. et al. North American ginseng (Panax quinquefolius) suppresses betaadrenergic-dependent signalling, hypertrophy, and cardiac dysfunction. Can. J. Physiol. Pharmacol. 94, 1325-1335 (2016).

47. Bertolotti, A., Zhang, Y., Hendershot, L. M., Harding, H. P. \& Ron, D. Dynamic interaction of BiP and ER stress transducers in the unfolded-protein response. Nat. Cell Biol. 2, 326-332 (2000)

48. Sawada, T. et al. X-box binding protein 1 regulates brain natriuretic peptide through a novel AP1/CRE-like element in cardiomyocytes. J. Mol. Cell. Cardiol. 48, 1280-1289 (2010).

49. Dally, S. et al. Compartmentalized expression of three novel sarco/endoplasmic reticulum $\mathrm{Ca}^{2+}$ ATPase 3 isoforms including the switch to ER stress, SERCA3f, in non-failing and failing human heart. Cell Calcium 45, 144-154 (2009).

50. $\mathrm{Fu}, \mathrm{H}$. Y. et al. Ablation of $\mathrm{C} / \mathrm{EBP}$ homologous protein attenuates endoplasmic reticulum-mediated apoptosis and cardiac dysfunction induced by pressure overload. Circulation 122, 361-369 (2010).
51. Sari, F. R. et al. Attenuation of CHOP-mediated myocardial apoptosis in pressure-overloaded dominant negative p38alpha mitogen-activated protein kinase mice. Cell. Physiol. Biochem. 27, 487-496 (2011).

52. Sreedhar, R. et al. Role of MAPK-mediated endoplasmic reticulum stress signaling in the heart during aging in senescence-accelerated prone mice. Biofactors 42, 368-375 (2016)

53. Pei, $\mathbf{H}$. et al. Notch1 cardioprotection in myocardial ischemia/reperfusion involves reduction of oxidative/nitrative stress. Basic Res. Cardiol. 108, 373 (2013).

54. Wang, J. et al. Induced overexpression of $\mathrm{Na}(+) / \mathrm{Ca}(2+)$ exchanger does not aggravate myocardial dysfunction induced by transverse aortic constriction. J. Card. Fail. 19, 60-70 (2013).

55. Lin, Y. et al. Critical role of astrocytic interleukin-17 A in post-stroke survival and neuronal differentiation of neural precursor cells in adult mice. Cell Death Dis. 7. e2273 (2016)

56. Zhai, M. et al. Melatonin protects against the pathological cardiac hypertrophy induced by transverse aortic constriction through activating PGC-1beta: in vivo and in vitro studies. J. Pineal Res. 63, https://doi.org/10.1111/jpi.12433 (2017).

57. Ruan, W. et al. MALAT1 up-regulator polydatin protects brain microvascular integrity and ameliorates stroke through C/EBPbeta/MALAT1/CREB/PGC1alpha/PPARgamma pathway. Cell. Mol. Neurobiol. https://doi.org/10.1007/ s10571-018-00646-4 (2019)

58. Dromparis, $\mathrm{P}$. et al. Attenuating endoplasmic reticulum stress as a novel therapeutic strategy in pulmonary hypertension. Circulation 127, 115-125 (2013).

59. Kojonazarov, B et al. p38 MAPK inhibition improves heart function in pressureloaded right ventricular hypertrophy. Am. J. Respir. Cell Mol. Biol. 57, 603-614 (2017). 